\title{
THE FIRST RELEASE COSMOS OPTICAL AND NEAR-IR DATA AND CATALOG ${ }^{1}$
}

\author{
P. Capak, ${ }^{2}$ H. Aussel, ${ }^{3,4}$ M. Ajiki, ${ }^{5}$ H. J. McCracken, ${ }^{3,6}$ B. Mobasher, ${ }^{7}$ N. Scoville,,${ }^{2,3}$ P. Shopbell, ${ }^{2}$ Y. Taniguchi, ${ }^{5,8}$ \\ D. Thompson, ${ }^{2,9}$ S. Tribiano, ${ }^{10,11}$ S. Sasaki, ${ }^{2,5,8}$ A. W. Blain, ${ }^{2}$ M. Brusa, ${ }^{12}$ C. Carilli, ${ }^{13}$ A. Comastri, ${ }^{14}$ \\ C. M. Carollo, ${ }^{15}$ P. Cassata, ${ }^{16}$ J. Colbert, ${ }^{17}$ R. S. Ellis, ${ }^{2}$ M. Elvis, ${ }^{18}$ M. Giavalisco, ${ }^{7}$ W. Green, ${ }^{2}$ \\ L. Guzzo, ${ }^{16}$ G. Hasinger, ${ }^{12}$ O. Ilbert, ${ }^{19}$ C. Impey, ${ }^{20}$ K. JahnKe, ${ }^{21}$ J. KartaltePe, ${ }^{19}$ J.-P. Kneib ${ }^{22}$ \\ J. Koda, ${ }^{2}$ A. KoeKemoer, ${ }^{7}$ Y. Komiyama, ${ }^{23}$ A. Leauthaud, ${ }^{2,22}$ O. Lefevre, ${ }^{22}$ S. Lilly, ${ }^{15}$ C. LiU, ${ }^{10}$ \\ R. Massey, ${ }^{2}$ S. Miyazaki, ${ }^{24}$ T. Murayama, ${ }^{5}$ T. Nagao, ${ }^{23,25}$ J. A. Peacock, ${ }^{26}$ A. Pickles, ${ }^{27}$ \\ C. Porciani, ${ }^{15}$ A. Renzini, ${ }^{28,29}$ J. Rhodes, ${ }^{2,30}$ M. Rich, ${ }^{31}$ M. Salvato, ${ }^{2}$ D. B. Sanders, ${ }^{19}$ \\ C. Scarlata, ${ }^{15}$ D. Schiminovich, ${ }^{32}$ E. Schinnerer, ${ }^{21}$ M. Scodeggio, ${ }^{33}$ K. Sheth, ${ }^{2,17}$ \\ Y. ShIOYA, ${ }^{8}$ L. A. M. TASCA, ${ }^{22}$ J. E. TAYlor, ${ }^{2}$ L. YAN, ${ }^{17}$ AND G. ZAMORANI ${ }^{34}$ \\ Received 2006 September 23; accepted 2007 April 6
}

\begin{abstract}
We present imaging data and photometry for the COSMOS survey in 15 photometric bands between 0.3 and $2.4 \mu \mathrm{m}$. These include data taken on the Subaru $8.3 \mathrm{~m}$ telescope, the KPNO and CTIO $4 \mathrm{~m}$ telescopes, and the CFHT $3.6 \mathrm{~m}$ telescope. Special techniques are used to ensure that the relative photometric calibration is better than $1 \%$ across the field of view. The absolute photometric accuracy from standard-star measurements is found to be $6 \%$. The absolute calibration is corrected using galaxy spectra, providing colors accurate to $2 \%$ or better. Stellar and galaxy colors and counts agree well with the expected values. Finally, as the first step in the scientific analysis of these data we construct panchromatic number counts which confirm that both the geometry of the universe and the galaxy population are evolving.
\end{abstract}

Subject headings: cosmology: observations — galaxies: evolution — large-scale structure of universe — surveys

\section{INTRODUCTION}

Advances in astronomy are often driven by improved accuracy and precision along with increases in sensitivity and area of the available data. The Canada-France Redshift Survey (CFRS;

\footnotetext{
1 Based in part on observations with the NASA/ESA Hubble Space Telescope, obtained at the Space Telescope Science Institute, which is operated by AURA, Inc., under NASA contract NAS5-26555; the Subaru telescope, which is operated by the National Astronomical Observatory of Japan; the MegaPrime/MegaCam, a joint project of CFHT and CEA/DAPNIA at the Canada-France-Hawaii Telescope, which is operated by the National Research Council of Canada, the Institute National des Science de l'Univers of the Centre National de la Recherche, and the University of Hawaii; and the Kitt Peak National Observatory, Cerro Tololo InterAmerican Observatory, and the National Optical Astronomy Observatory, which is operated by the Association of Universities for Research in Astronomy, Inc., under cooperative agreement with the National Science Foundation.

2 California Institute of Technology, MC 105-24, 1200 East California Boulevard, Pasadena, CA 91125.

3 Visiting Astronomer, University of Hawaii, 2680 Woodlawn Drive, Honolulu, HI 96822.

4 AIM, Unité Mixte de Recherche, UMR 7158, CNRS, Université Paris VII, CEA/Saclay, F-91191 Gif-sur-Yvette, France.

5 Astronomical Institute, Graduate School of Science, Tohoku University, Aramaki, Aoba, Sendai 980-8578, Japan.

6 Institut d'Astrophysique de Paris, UMR 7095, CNRS, Université Pierre et Marie Curie, 98 bis Boulevard Arago, F-75014 Paris, France.

7 Space Telescope Science Institute, 3700 San Martin Drive, Baltimore, MD 21218.

8 Physics Department, Graduate School of Science and Engineering, Ehime University, 2-5 Bunkyo-cho, Matsuyama 790-8577, Japan.

9 LBT Observatory, University of Arizona, 933 North Cherry Avenue, Tucson, AZ 85721-0065.

10 American Museum of Natural History, Central Park West at 79th Street, New York, NY 10024.

11 CUNY Borough of Manhattan Community College, 199 Chambers Street, New York, NY 10007.

12 Max-Planck-Institut für Extraterrestrische Physik, D-85478 Garching, Germany.

13 National Radio Astronomy Observatory, P.O. Box O, Socorro, NM 87801-0387.

14 INAF-Osservatorio Astronomico di Bologna, via Ranzani 1, I-40127 Bologna, Italy.

${ }^{15}$ Department of Physics, ETH Zurich, CH-8093 Zurich, Switzerland.
}

Lilly et al. 1995), the Hawaii Deep Surveys (Cowie et al. 1999), and the Hubble Deep Fields (HDFs; Williams et al. 1996; Casertano et al. 2000) were the first deep imaging and spectroscopic surveys aimed at understanding galaxy formation and evolution. These discovered the global decline in star formation at

16 INAF-Osservatorio Astronomico di Brera, via Bianchi 46, I-23807 Merate (LC), Italy.

17 Spitzer Science Center, California Institute of Technology, Pasadena, CA 91125.

${ }^{18}$ Harvard-Smithsonian Center for Astrophysics, 60 Garden Street, Cambridge, MA 02138

19 Institute for Astronomy, 2680 Woodlawn Drive, University of Hawaii, Honolulu, HI 96822.

20 Steward Observatory, University of Arizona, 933 North Cherry Avenue, Tucson, AZ 85721.

21 Max-Planck-Institut für Astronomie, Königstuhl 17, D-69117, Heidelberg, Germany.

${ }^{22}$ Laboratoire d'Astrophysique de Marseille, BP 8, Traverse du Siphon, F-13376 Marseille Cedex 12, France.

23 National Astronomical Observatory of Japan, 2-21-1 Osawa, Mitaka, Tokyo 181-8588, Japan.

${ }^{24}$ Subaru Telescope, National Astronomical Observatory of Japan, 650 North A'ohoku Place, Hilo, HI 96720.

25 INAF-Osservatorio Astrofisico di Arcetri, Largo Enrico Fermi 5, I-50125 Firenze, Italy.

${ }^{26}$ Institute for Astronomy, University of Edinburgh, Royal Observatory, Blackford Hill, Edinburgh EH9 3HJ, UK.

27 Caltech Optical Observatories, MS 320-47, California Institute of Technology, Pasadena, CA 91125.

${ }^{28}$ European Southern Observatory, Karl-Schwarzschild-Strasse 2, D-85748 Garching, Germany.

29 Dipartimento di Astronomia, Université di Padova, Vicolo dell'Osservatorio 2, I-35122 Padua, Italy.

30 Jet Propulsion Laboratory, Pasadena, CA 91109.

31 Department of Physics and Astronomy, University of California, Los Angeles, CA 90095.

32 Department of Astronomy, Columbia University, MC 2457, 550 West 120th Street, New York, NY 10027.

${ }^{33}$ INAF-IASF Milano, via Bassini 15, I-20133 Milano, Italy.

34 INAF-Osservatorio Astronomico di Bologna, via Ranzani 1, I-40127 Bologna, Italy. 
TABLE 1

Telescopes Used for COSMOS Optical/IR Data in 2005-2006

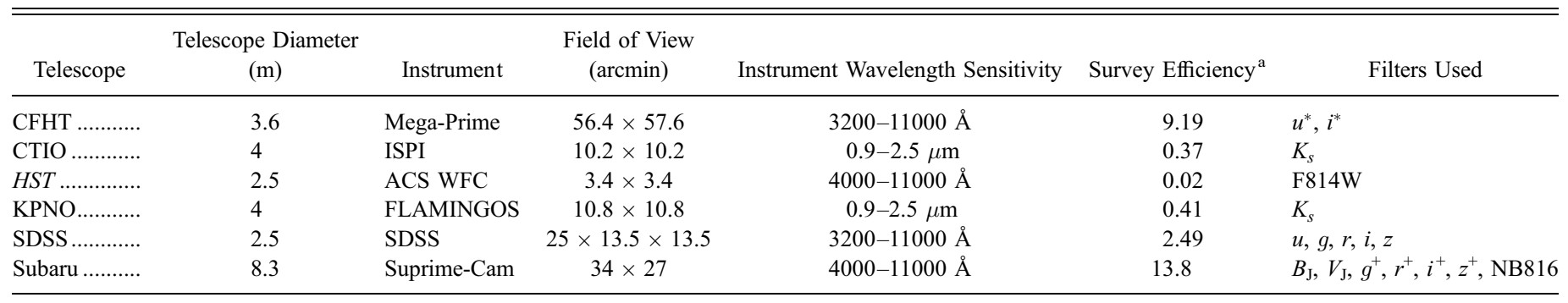

${ }^{\text {a }}$ Defined as the telescope collecting area multiplied by the imaging area in square degrees.

$z<1$ and showed that this was due to star formation occurring in smaller galaxies at later times (Lilly et al. 1996; Cowie et al. 1999), a phenomenon often referred to as "cosmic downsizing." At the same time Steidel et al. $(1996,1999,2003)$ used the Lyman break galaxy color selection technique to identify galaxies at high redshift, dramatically improving the efficiency of spectroscopic surveys at $z>3$. Other selections such as the $B z K$ (Daddi et al. 2004), BX/BM (Adelberger et al. 2004), and distant red galaxy selections (Franx et al. 2003) have allowed for efficient sorting of $1<z<3$ galaxies.

Photometric redshifts are the logical extension of color selection by estimating redshifts and spectral energy distributions (SEDs) from many photometric bands. Unlike color selection, photometric redshifts take advantage of all available information, enabling redshift estimates along with the age, star formation rate, and mass. Unfortunately, photometric redshifts are also susceptible to systematics in all bands. This increases the calibration requirements, especially the required photometric accuracy, for modern cosmological surveys such as the Great Observatories Origins Deep Survey (GOODS; Giavalisco et al. 2004), the Galaxy Evolution from Morphology and SEDs (GEMS) survey (Rix et al. 2004), and the Cosmic Evolution Survey, or COSMOS (Scoville et al. 2007b).

GOODS and GEMS are designed to study the evolution of galaxies with look-back time, whereas COSMOS is designed to probe the evolution of galaxies in the context of their large-scale structure out to moderate redshift. The desire to study large-scale structure in COSMOS necessitates a $2 \mathrm{deg}^{2}$ area with deep panchromatic data. Such data have been collected at nearly every observable wavelength from the X-rays to the radio. The study of large-scale structures places strong calibration requirements on the COSMOS data; for example, spatial variations in photometry and astrometry must be kept to a minimum, typically less than $1 \%$ for photometry, to ensure high-quality photometric redshifts and $0.01^{\prime \prime}$ positional accuracy for astrometry to enable measurements of weak lensing and correlation functions. Meeting these calibration requirements is often difficult, as multiple instrument pointings are used to cover the field.

This paper concentrates on the ground-based data reduction, the multiband optical and near-infrared catalog, and the steps taken to ensure a high level of photometric consistency. The observing strategy for the Subaru Suprime-Cam observations, which form the bulk of our ground-based data, are discussed separately in Taniguchi et al. (2007). In addition, the absolute photometric and astrometric system used here is defined in H. Aussel et al. (2007, in preparation).

An overview of the COSMOS project and its goals are given in Scoville et al. (2007b). Details of the Hubble Space Telescope (HST) observations, including the Advanced Camera for Surveys (ACS), the Wide Field Planetary Camera 2 (WFPC2), and the
Near Infrared Camera and Multi-Object Spectrometer (NICMOS) are found in Scoville et al. (2007a). The ACS data acquisition and reduction are detailed in Koekemoer et al. (2007), and a monochromatic catalog based only on the HST ACS observations is presented in Leauthaud et al. (2007). Observations at other wavelengths consist of X-ray observations with XMM-Newton (Hasinger et al. 2007), ultraviolet observations with GALEX (Zamojski et al. 2007), mid-infrared observations with the Spitzer Space Telescope (Sanders et al. 2007), submillimeter observations from the Caltech Submillimeter Observatory (CSO; Aguirre et al. 2007) and the Institut de Radioastronomie Millimétrique (IRAM) $30 \mathrm{~m}$ telescope (Bertoldi et al. 2007), and radio observations with the Very Large Array (VLA; Schinnerer et al. 2004, 2007).

We begin by presenting an overview of the various data sets and photometric systems, the imaging data products, and the data reduction in $\S 2$. Point-spread function (PSF) matching is covered in $\S 3$, and the generation of a multicolor catalog is presented in $\S 4$. Finally, in $\S 5$ we conduct several quality checks, and suggest several corrections to the absolute photometry.

\section{OBSERVATIONS AND DATA REDUCTION}

The present COSMOS data were collected on a variety of telescopes and instruments, as well as from the Sloan Digital Sky Survey (SDSS) second data release (DR2) archive (Abazajian et al. 2004). This paper covers the processing of the data obtained with Suprime-Cam (Komiyama et al. 2003) on the Subaru $8.3 \mathrm{~m}$ telescope, Megaprime (Aune et al. 2003; Boulade et al. 2003) on the 3.6 m Canada-France-Hawaii Telescope (CFHT), FLAMINGOS (Elston 1998) on the Kitt Peak National Observatory (KPNO) $4 \mathrm{~m}$ telescope, and the Infrared Side Port Imager (ISPI; Probst et al. 2003) on the Cerro Tololo Inter-American Observatory (CTIO) $4 \mathrm{~m}$ telescope during the 2004-2005 observing season.

The telescopes and instruments used for the COSMOS survey are presented in Table 1. A survey efficiency is given for each telescope-instrument pair to allow comparisons between the various data sets. The survey efficiency is defined as the telescope collecting area multiplied by the detector imaging area $\left(\mathrm{deg}^{2} \mathrm{~m}^{2}\right)$ and does not include variations in detector sensitivity, sky background, or field geometry. This number is most useful for comparing observations taken in similar bands. The filter transmission profiles, including atmospheric transmission, telescope reflectivity, instrument optical transmission, filter transmission, and detector sensitivity, are plotted in Figures 1 and 2 in units of relative detector quantum efficiency normalized to 1 at the peak.

The Suprime-Cam, Megaprime, SDSS photometric, and SDSS survey cameras have filters distinct from each other and from the Landolt standard star system. Even the SDSS photometric telescope and SDSS survey telescope filter sets differ from one another by $2 \%-4 \%$. To differentiate between these filter systems we 


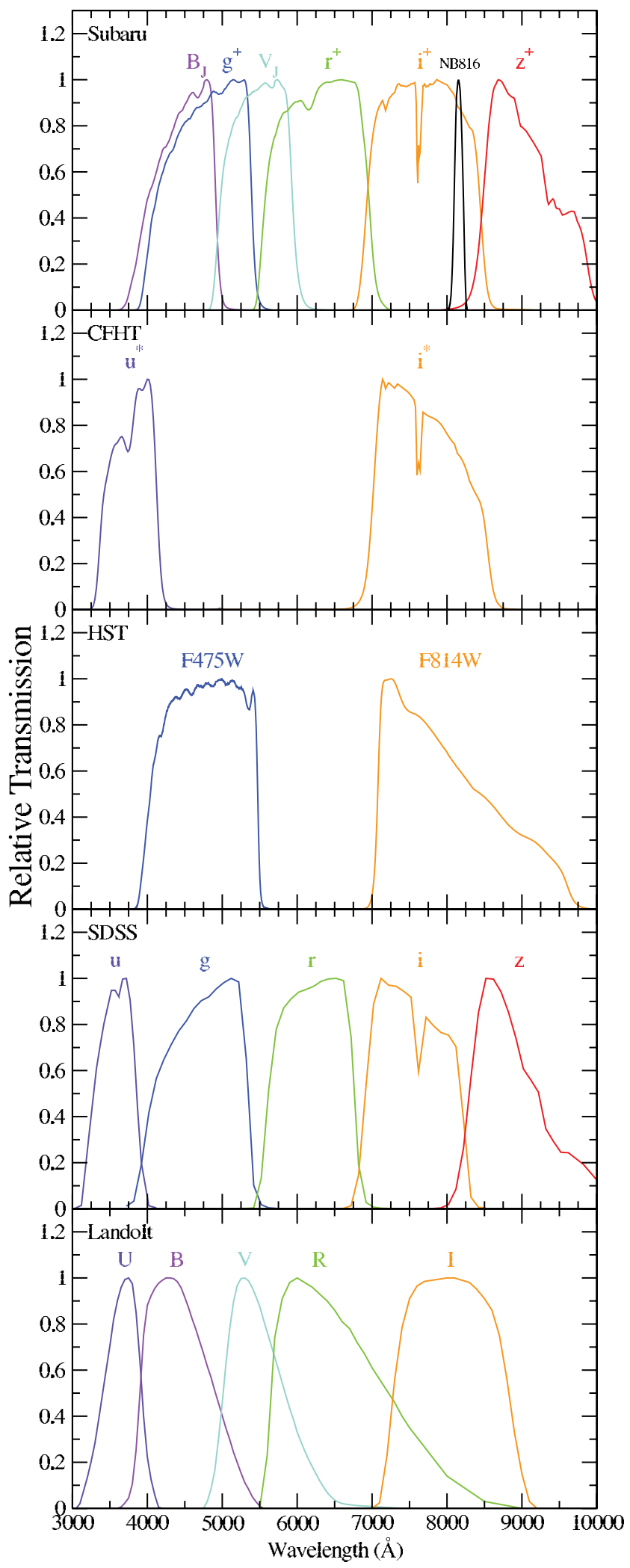

Fig. 1.-Filter transmission profiles for the COSMOS optical data set from CFHT, Subaru, and HST as of 2005 April. These profiles are normalized to a maximum throughput of 1 and include the transmission of the atmosphere, the telescope, the camera optics, the filter, and the detector. The HST F475W data only cover the central $9^{\prime} \times 9^{\prime}$ area; details are given in Scoville et al. (2007a). The SDSS Abazajian et al. (2004) and Johnson-Cousins systems used by Landolt (1992) are shown for comparison. Notice the significant differences between the Johnson-Cousins, SDSS, and other systems. Color conversions are clearly needed to transform from the COSMOS system to the standard star systems. These are given in $\mathrm{H}$. Aussel et al. (2007, in preparation).

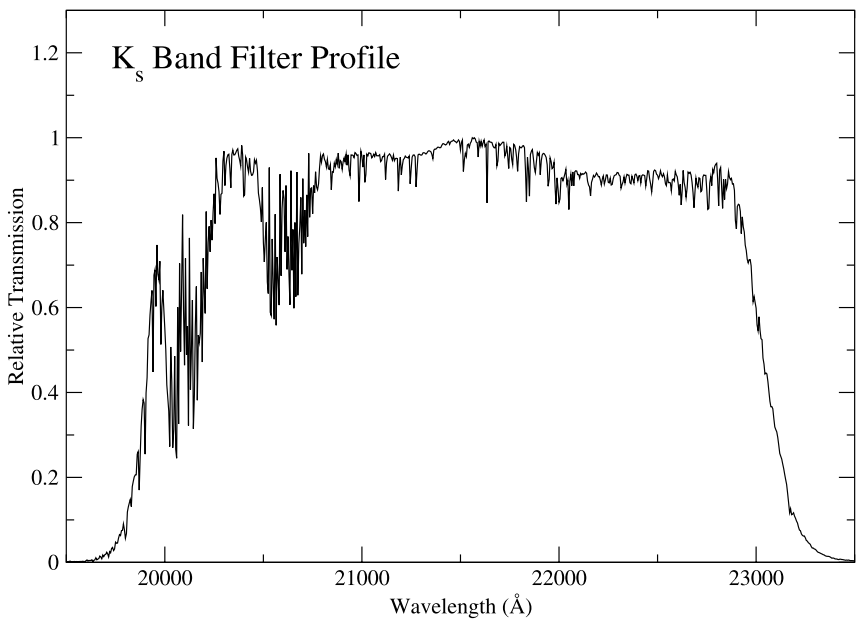

FIG. 2.-Transmission profile of the $K_{\mathrm{s}}$-band filter from KPNO FLAMINGOS and CTIO ISPI. This profile is normalized to a maximum throughput of 1 and includes the transmission of the atmosphere, the telescope, the camera optics, the filter, and the detector.

use a plus sign superscript for the Suprime-Cam Sloan filters and an asterisk superscript for the Megaprime Sloan filters; no superscript is used for the SDSS survey filters. The designations $U, B, V$, $R$, and $I$ are used for the Landolt-Johnson-Cousins set, while $B_{\mathrm{J}}$ and $V_{\mathrm{J}}$ are used for the Suprime-Cam Johnson set. Conversions between these systems are discussed in H. Aussel et al. (2007, in preparation).

The wavelength range, depth, and image quality for all data presently reduced and included in the version 2.0 optical/IR catalog are given in Table 2 and plotted in Figure 3. The depth quoted is for a $5 \sigma$ measurement in a $3^{\prime \prime}$ aperture of an isolated point source at the median seeing given in Table 2. This should be viewed as an optimistic estimate since most objects are extended and many are confused with neighboring sources. Taniguchi et al. (2007) present a discussion of detection sensitivities and completeness for various Subaru filters. The median photometric depths in the COSMOS $i^{+}$ selected catalog are discussed in $\S 4$. Table 2 also gives a first-order offset to the Vega system; however, a color term must be applied to get the true Landolt-Vega system magnitudes; these are given in H. Aussel et al. (2007, in preparation).

\subsection{Data Products}

We took special care in producing data products that simplify analysis and are tractable on contemporary computers. ${ }^{35}$ To do this we defined a common grid of subimages for all data products. The starting point for this grid is the COSMOS astrometric catalog, which covers $4 \operatorname{deg}^{2}$ (H. Aussel et al. 2007, in preparation) and is larger than all present or planned COSMOS data sets. The area is divided into 144 sections of $10^{\prime} \times 10^{\prime}$, and each section is covered by an image of size $4096 \times 4096$ pixels with a pixel scale of $0.15^{\prime \prime}$. Therefore, adjacent tiles overlap each other by $14.4^{\prime \prime}$ on all sides. As a result, the vast majority of objects can be analyzed on a single image. The layout of the image tiles is shown in Figure 4. The pixel scale was chosen to be an integer multiple of the $0.05^{\prime \prime}$ scale used for the HST ACS images. All images and noise maps are scaled to units of nanojanskys per pixel, which corresponds to a magnitude zero point of 31.4.

For each Subaru and SDSS band an image with the original PSF and a PSF-homogenized across the field within that band is

35 All data products discussed in this paper are publicly available at http:// irsa.ipac.caltech.edu/data/COSMOS/. 
TABLE 2

Data Quality and Depth

\begin{tabular}{|c|c|c|c|c|c|c|}
\hline Filter Name & $\begin{array}{c}\text { Central Wavelength } \\
(\AA)\end{array}$ & $\begin{array}{l}\text { Filter Width } \\
(\AA)\end{array}$ & $\begin{array}{l}\text { Seeing Range } \\
\text { (arcsec) }\end{array}$ & Depth $^{\mathrm{a}, \mathrm{b}}$ & Saturation Magnitude $^{\mathrm{b}}$ & Offset from Vega System $^{c}$ \\
\hline 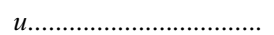 & 3591.3 & 550 & $1.2-2.0$ & 22.0 & 12.0 & 0.921 \\
\hline$B_{\mathrm{J}} \ldots \ldots \ldots \ldots \ldots \ldots \ldots \ldots$ & 4459.7 & 897 & $0.4-0.9$ & 27.3 & 18.7 & -0.131 \\
\hline$g$ & 4723.1 & 1300 & $1.2-1.7$ & 22.2 & 12.0 & -0.117 \\
\hline$g^{+} \ldots \ldots \ldots \ldots \ldots \ldots \ldots \ldots \ldots \ldots$ & 4779.6 & 1265 & $0.7-2.1$ & 27.0 & 18.2 & -0.117 \\
\hline$V_{\mathrm{J}} \ldots \ldots \ldots \ldots \ldots \ldots \ldots \ldots$ & 5483.8 & 946 & $0.5-1.6$ & 26.6 & 18.7 & -0.004 \\
\hline$i$ i. & 7522.5 & 1300 & $0.9-1.7$ & 21.3 & 12.0 & 0.355 \\
\hline$i^{+} \ldots \ldots \ldots \ldots \ldots \ldots \ldots \ldots \ldots \ldots \ldots$ & 7640.8 & 1497 & $0.4-0.9$ & 26.2 & $20.0^{\mathrm{d}}$ & 0.379 \\
\hline$i^{*}$ & 7683.6 & 1380 & 0.94 & 24.0 & 16.0 & 0.380 \\
\hline 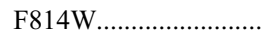 & 8037.2 & 1862 & 0.12 & $24.9^{\mathrm{e}}$ & 18.7 & 0.414 \\
\hline NB816 ................................. & 8151.0 & 117 & $0.4-1.7$ & 25.7 & 16.9 & 0.458 \\
\hline$z$ & 8855.0 & 1000 & $1-1.7$ & 20.5 & 12.0 & 0.538 \\
\hline
\end{tabular}

${ }^{\text {a }}$ This is $5 \sigma$ in a $3^{\prime \prime}$ aperture for an isolated point source at the native seeing.

${ }^{\mathrm{b}}$ In $\mathrm{AB}$ magnitudes.

${ }^{c}$ AB magnitude $=$ Vega magnitude + offset. This offset does not include the color conversions to the Johnsons-Cousins system used by Landolt (1992).

${ }^{\mathrm{d}}$ Compact objects saturate at $i^{+}<21.8$ due to the exceptional seeing.

e The sensitivity for photometry of an optimally extracted point source is 27.1 ; for optimal photometry of a $1^{\prime \prime}$ galaxy it is 26.1 .

provided. For the Subaru $B_{\mathrm{J}}, r^{+}$, and $i^{+}$bands, which have exceptional image quality $\left(0.5^{\prime \prime}-0.8^{\prime \prime}\right.$ seeing), a "best seeing" image is also provided. The CFHT images were taken in queue observing mode, ensuring a consistent PSF for all observations, so only an original PSF image is provided for these data. Finally, due to the large variation of the PSF in the CTIO and KPNO data, only a PSF-homogenized image is provided.

In addition, rms noise maps are provided for each filter. These are on the same tiling scheme and flux scale as the images. The rms maps include noise contributions from photon noise, background subtraction, flat-fielding, defect masking, saturation, and cosmic-ray removal. They do not include the photon noise contribution from object flux.

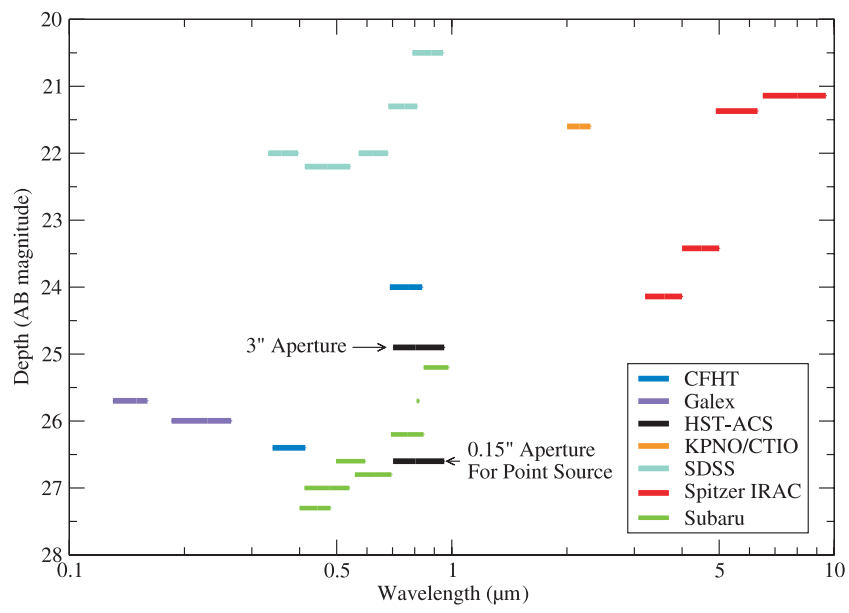

Fig. 3.-Background-limited depth of COSMOS observations in the ultraviolet, optical, and infrared. The CFHT, KPNO/CTIO, and Subaru depths are $5 \sigma$ in a $3^{\prime \prime}$ aperture. The SDSS depths are those quoted in Abazajian et al. (2004). The depth of the HST ACS observations is given for a $3^{\prime \prime}$ aperture and a $0.15^{\prime \prime}$ aperture with a point source. A $3^{\prime \prime}$ aperture is optimal for color measurements, while the $0.15^{\prime \prime}$ aperture is the $5 \sigma$ detection limit for point sources. The Spitzer IRAC depths are those expected at $5 \sigma$ in a $3^{\prime \prime}$ aperture for observations taken in 2006. The GALEX depths are from Zamojski et al. (2007).

\subsection{Subaru Suprime-Cam}

The Suprime-Cam instrument (Komiyama et al. 2003) on the Subaru $8.3 \mathrm{~m}$ telescope has a $34^{\prime} \times 27^{\prime}$ field of view. The camera has $102 \mathrm{~K} \times 4 \mathrm{~K}$ Lincoln Labs $\mathrm{CCD}$ detectors, which have good sensitivity between 4000 and $10000 \AA$. Nine Suprime-Cam pointings were required to cover the COSMOS field. During 2004 and 2005, data were obtained in the $B_{\mathrm{J}}, V_{\mathrm{J}}, g^{+}, r^{+}, i^{+}$, and $z^{+}$ broadband and the NB816 narrowband filters. These SuprimeCam observations, which required special planning, are detailed in Taniguchi et al. (2007). Further observations in $11300 \AA$ intermediate bands, IA427, IA464, IA484, IA505, IA527, IA624, IA679, IA709, IA738, IA767, and IA827, and one narrow band, NB711, were obtained in 2006 and 2007. These new observations

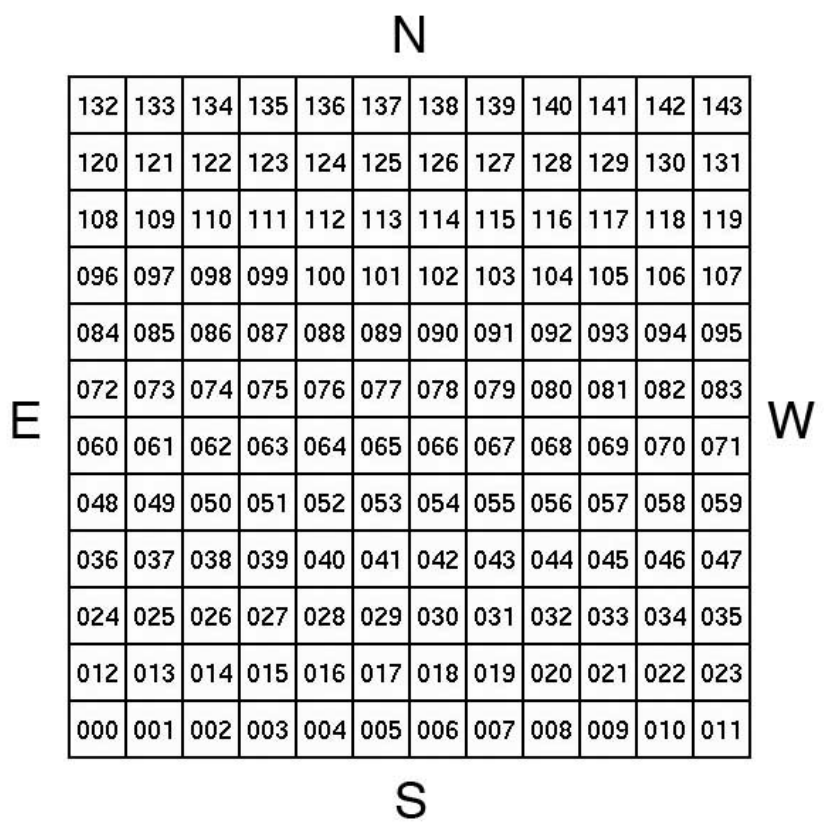

FIG. 4.-Layout of image tiles for the COSMOS field. 
have been reduced using the prescription described here, but will be presented elsewhere.

Objects brighter than 19 mag are saturated in a typical exposure, and under good seeing the saturation level can drop to $22 \mathrm{mag}$ in long exposures. As a result, it is extremely difficult to astrometrically calibrate these data against external astrometric catalogs such as the SDSS (Abazajian et al. 2004) and USNO-B1.0 (Monet et al. 2003), which only reach $21 \mathrm{mag}$. To mitigate this limitation, a series of short exposures were taken in each band.

\subsubsection{Initial Calibration}

The first step in our Suprime-Cam data reduction is to measure a bias level from the overscan region and subtract it from all the images. Then all bad or saturated pixels are masked. Next, a median bias frame is constructed from overscan-corrected frames. Following the overscan correction, this bias frame is subtracted from data and flat frames in order to remove bias structures. In particular, the bias level increases near the edges of the CCDs farthest from the readout register.

A median dome flat for each band is then constructed from 10-20 bias-subtracted flat-field images. The median dome flats and median biases are inspected for bad pixels, charge traps, and other defects that need to be masked. The appropriate median dome flat, with all defects masked, is then used to normalize all data frames. Finally, portions of the image vignetted by the guide probe are masked from all data frames using the position of the guide probe recorded in the image header.

After the initial calibration catalogs are generated for every data frame with the IMCAT ${ }^{36} \mathrm{hf}$ indpeaks and apphot routines using $5^{\prime \prime}$ diameter apertures. The large aperture is chosen to minimize photometric variations caused by changes in seeing. This catalog is then used to generate an object mask for each frame and to calculate the astrometric solution.

The night sky is subtracted in a two-step process to account for fringing and scattered light. First, a normalized median sky frame is constructed for each night. The median frame is generated by masking objects in all frames, normalizing every frame to the same median flux, and finally median-combining the normalized images. The median sky is then scaled to the median background level in each frame and subtracted. This removes both night sky illumination and fringing.

After subtracting the median sky, residual scattered light is visible on the images. This residual light affects both the overall flat field and the background of each individual frame. A correction to the flat field is described in $\S 2.2 .2$, while the light in each frame is subtracted by masking objects and measuring the median of the residual background in $128 \times 128$ pixel squares. A background image is then generated by tesselating over the grid of medians. After subtracting this background, no visible sky structure is left on the individual frames. However, this step creates negative halos around bright stars and very extended galaxies due to imperfect masking. Fortunately, the amplitude of these halos is similar in all frames and can be accounted for as a residual background in the combined images.

After sky subtraction, an astrometric solution is calculated separately for all exposures and CCDs by matching the object catalogs to the COSMOS astrometric catalog (H. Aussel et al. 2007, in preparation) using a fourth-order, two-dimensional polynomial. The polynomial fits are improved by removing mismatched objects in an iterative fashion until the solution converges (typically in two iterations). The resulting scatter between the fit positions

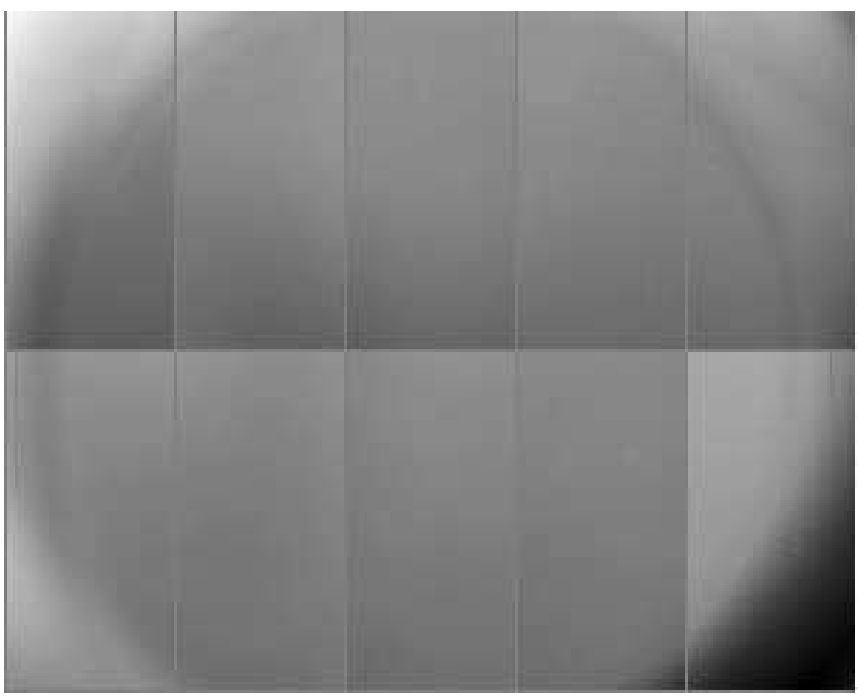

FIG. 5.-Difference between Suprime-Cam dome flats taken in $r^{+}$at position angles of $0^{\circ}$ and $+90^{\circ}$ with chip-to-chip sensitivity variations removed. The scale is linear with a stretch of $-3 \%$ to $+3 \%$ from black to white. Variations in the illumination pattern due to scattered light are clearly visible.

and the final astrometry is always less than $0.2^{\prime \prime}$ at the $1 \sigma$ level, independent of position.

Using the astrometric solutions, defects around charge bleeds from saturated stars are masked using a list of bright stars from the SDSS and the USNO-B1.0. Cosmic-ray events are removed by detecting sharp edges in the images. Finally, every frame is visually inspected to remove internal reflections, satellites, asteroids, and other false objects. Once all masking is complete, a new photometric catalog is generated containing only isolated objects in unmasked regions.

\subsubsection{Scattered-Light Correction}

Mechanical and optical constraints make it impossible to baffle wide-field cameras against all scattered light. The scattered light is equivalent to an unknown dark current added to each image, and must be subtracted rather than divided out. As a result, the usual flat-fielding technique of observing a uniform light source such as the dome or sky is inaccurate at the $3 \%-5 \%$ level.

For Suprime-Cam the scattered-light pattern and strength change significantly with the lighting conditions and telescope position. Variations as large as $\pm 5 \%$ are observed at the edges of the field between dark, twilight, and dome conditions. Figure 5 shows the difference between two dome flats taken at different rotation angles. This effect is similar in amplitude and pattern to that observed with the $12 \mathrm{~K}$ and Megacam cameras on the CFHT. $^{37}$

Following the example of CFHT we calculate the true flat by observing objects at multiple positions on the camera. The true flat can then be solved for as the flat field, which yields the same background-subtracted flux for an object at any position in the field of view. In practice the flat image is generated by dividing the focal plane into $128 \times 128$ pixel regions $r$, and calculating a factor $C_{r}$ for each region. The regions are defined so that no region crosses a detector boundary. As a result, sensitivity variations due to detectors are also measured. We can also allow for an additional factor $P_{e}$ between exposures to correct for photometric variations due to atmospheric conditions and seeing. 
However, if the data are photometric and corrected for air mass, $P_{e}=0$ for all exposures.

For a single object the real magnitude $M_{\text {real }}$ is described by

$$
M_{\text {real }}=M+C_{r}+P_{e},
$$

where $M$ is the measured magnitude. If we consider a pair of exposures, $a$ and $b$, we can construct a $\chi^{2}$ relation as

$$
\chi^{2}=\sum_{a=0}^{N \exp } \sum_{b=a+1}^{N \exp } \sum_{i=o}^{N o b j} \frac{\left(M_{i, a}-M_{i, b}+C_{r, a}-C_{r, b}+P_{a}-P_{b}\right)^{2}}{\sigma_{i, a}^{2}+\sigma_{i, b}^{2}}
$$

which can be minimized to obtain the $C_{r}$ and $P_{e}$ factors. Since an object can only belong to one region in each exposure we use the notation $C_{r, a}$ to indicate the region an object belongs to in exposure $a$.

To ensure that equation (2) is well constrained, a series of 24 short exposures were taken in each band in each of two orientations. Each exposure overlapped its neighbors by $50 \%$. As a result, any given point in the COSMOS field was observed by at least eight different areas of Suprime-Cam. These data were calibrated and the photometry performed as described in $\S 2.2 .1$. The photometry was then corrected for variations in pixel area using the Jacobian of the optical distortion. This corrected for the assumption that all pixels have the same size when the dome flat is applied. Each object was then assigned a unique ID by merging the catalogs from each exposure with the master astrometry catalog. The IMCAT routine fitmagshifts was then used to solve equation (2) for the $P_{e}$ and $C_{r}$ factors.

Two flat fields were generated for each band; all the data were used in one, and only the data with high photometric quality were in the other. No significant difference was observed between these flats in any band. As a result we included all the data in our solutions. This provides an additional check on the photometric calibration because an air-mass term can be calculated independently from the data and from the standard stars. In addition, nonphotometric data can be properly corrected for extinction.

The 24 short exposures were taken while the camera was under similar but opposite gravity loads for each orientation. The flat fields measured from these two orientations agree to within $1 \%$, ruling out mechanical flexure as a significant source of calibration error. Exposures were also taken in the same band over several runs to test for changes in the flat field due to instrument movements. No significant changes are seen in the flat field between runs spaced as much as a year apart. In contrast, the scattered-light pattern, and hence the dome flat, changed by as much as $2 \%$.

Since no variations were observed in the scattered-lightcorrected flat field as a function of telescope position, time, or photometric quality, the same flat can be and was used for multiple telescope runs. These flats are publicly available as part of the COSMOS archive. An example of the correction for the $r^{+}$ band is shown in Figure 6.

We find that the scattered-light component is largest in the outer $8^{\prime}$ of the field of view, which is vignetted by the primary mirror. In this outer region the typical correction to a dome-flattened image is $2 \%-4 \%$. In contrast, the central $26^{\prime}$ of the field of view is stable and flat to $1 \%$ with no calibration. Therefore, the scattered light can be safely ignored for the inner regions of Suprime-Cam.

\subsubsection{Photometric Calibration and Image Combination}

After flat-fielding, object frames taken on photometric nights are corrected for atmospheric extinction measured from standard

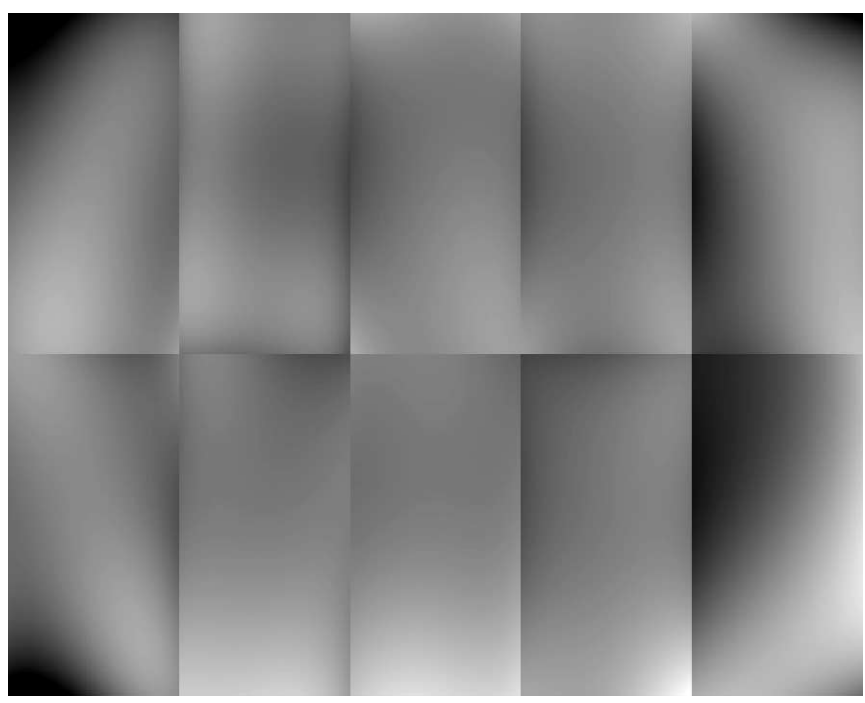

FIG. 6.-Relative correction to the $r^{+}$Suprime-Cam dome flat with chip-tochip sensitivity variations removed. The scale is linear with a stretch of $-3 \%$ to $+3 \%$ from black to white. A correction for scattered light in the vignetted portion of the field is clearly visible around the edge of the field of view.

stars (H. Aussel et al. 2007, in preparation). Data taken on nonphotometric nights are scaled to those taken in photometric conditions using the $P_{e}$ factors calculated during the scattered-light correction. Exposures with extinction greater than 0.5 mag are discarded. Absolute photometric calibration is done on the AB system using the Subaru filter transmission curves. As a result, all images are in units of nanojanskys per pixel. Color conversion and methodology are discussed in H. Aussel et al. (2007, in preparation).

After calibration the images in any given band are smoothed to the same full width at half-maximum (FWHM) using a Gaussian kernel. They are then resampled onto the final astrometric grid with a sum-over-triangles interpolation using the IMCAT warpimage task. Inverse-variance maps, derived from the image noise and flat fields, are also generated and resampled onto the final astrometric grid. The variance is scaled so that the noise measured in a given sky area on the resampled images is identical to that measured in the same area on the original images.

Once resampled, the images and variance maps are combined with the IMCAT combineimages command using a weighted sum with outlier pixels, more than $5 \sigma$ from the median, removed. A final rms map is also generated by combineimages that reflects the true pixel-to-pixel rms. The PSF-homogenized images provide the most consistent photometry but lose some sensitivity due to the smoothing.

A second combination was done with the original PSF images for all bands to provide a maximum-sensitivity image for detection. Since the PSF varies as a function of position and magnitude in these images, the aperture photometry and colors are less reliable. Finally, for the $B_{\mathrm{J}}, r^{+}$, and $i^{+}$data a third combination of the images was done with only the best seeing data. The resulting images have FWHMs of $0.6^{\prime \prime}, 0.8^{\prime \prime}$, and $0.5^{\prime \prime}$, respectively.

\subsection{CFHT Megaprime}

The Megaprime camera has a $1 \mathrm{deg}^{2}$ field of view on the $3.6 \mathrm{~m}$ CFHT. The focal plane is covered with $362 \mathrm{~K} \times 4.5 \mathrm{~K}$ EEV CCD detectors with excellent response between 3200 and $9000 \AA$ (Aune et al. 2003; Boulade et al. 2003).

Megaprime was used to obtain deep $u^{*}$-band (3798 $\AA$ ) and shallow $i^{*}$-band images of the COSMOS field. Objects as bright 
TABLE 3

Summary Observing Log for CFHT

\begin{tabular}{|c|c|c|}
\hline Filter & $\begin{array}{l}\text { Exposure Time } \\
\text { (hr) }\end{array}$ & Observation Date \\
\hline \multirow[t]{22}{*}{$u^{*}$} & 1.8 & 2003 Dec 21 \\
\hline & 1.8 & 2003 Dec 22 \\
\hline & 1.9 & 2004 Jan 19 \\
\hline & 3.4 & 2004 Jan 20 \\
\hline & 1.8 & 2004 Apr 22 \\
\hline & 1.8 & 2004 Apr 25 \\
\hline & 0.6 & 2004 May 22 \\
\hline & 0.3 & 2005 Apr 4 \\
\hline & 0.2 & 2005 Apr 5 \\
\hline & 1.5 & 2005 Apr 9 \\
\hline & 1.0 & 2005 Apr 11 \\
\hline & 1.0 & 2005 Apr 14 \\
\hline & 1.0 & 2005 May 4 \\
\hline & 0.9 & 2005 May 5 \\
\hline & 0.9 & 2005 May 6 \\
\hline & 0.9 & 2005 May 8 \\
\hline & 0.4 & 2005 May 29 \\
\hline & 0.4 & 2005 Jun 2 \\
\hline & 0.2 & 2005 Jun 3 \\
\hline & 0.4 & 2005 Jun 4 \\
\hline & 0.4 & 2005 Jun 5 \\
\hline & 0.2 & 2005 Jun 6 \\
\hline \multirow[t]{4}{*}{$i^{*} \ldots \ldots \ldots \ldots \ldots \ldots \ldots \ldots \ldots \ldots \ldots \ldots \ldots \ldots \ldots$} & 0.1 & 2003 Dec 21 \\
\hline & 0.9 & 2004 Jan 15 \\
\hline & 1.3 & 2004 Jan 17 \\
\hline & 2.9 & 2004 Jan 18 \\
\hline
\end{tabular}

as 15 mag are unsaturated in long exposures with Megaprime, allowing for an excellent astrometric solution. Observations were taken in sets of five dithered exposures forming a five-point dieface pattern. A total of five overlapping pointing centers were observed to cover the COSMOS field, resulting in data 4 times deeper in the center of the field than on the edges. The depth variation is recorded in the noise maps discussed in $\S 2.1$. A summary of the observing logs for the Megaprime observations is given in Table 3.

The CFHT operates in a queue observing mode for Megaprime observations, which ensures uniform image quality and photometry. CFHT and TERAPIX also provide a standard reduction pipeline, which meets our calibration requirements.

Appropriate calibration frames were taken each night by the queue observer, and calibrated data were provided by the Elixir pipeline (Magnier \& Cuillandre 2004). This pipeline corrects for bias, dark current, flat-fielding, and scattered light, with the final photometric calibration better than $1 \%$ across the field of view.

Further reduction including astrometric and photometric calibration, sky subtraction, and image combination was provided by the TERAPIX data-processing center. ${ }^{38}$ At TERAPIX the calibrated images provided by Elixir were visually inspected with the qualityFITS data quality assessment tool, and any defective images were rejected. Images with a seeing larger than $1.3^{\prime \prime}$ in the $i^{*}$ band and $1.4^{\prime \prime}$ in the $u^{*}$ band were also rejected.

All of the images were astrometrically registered to the COSMOS catalog (H. Aussel et al. 2007, in preparation) using the Astrometrix package. They were then resampled using a Lancsos-3 interpolation kernel and median-combined using the SWarp image combination software. Although the median combination is suboptimal in signal-to-noise ratio, it provides the best rejection of cosmic rays. The rms maps, derived from the image noise and

\footnotetext{
${ }^{38}$ See http://terapix.iap.fr/soft/.
}

TABLE 4

Summary Observing Log for CTIO and KPNO

\begin{tabular}{|c|c|c|c|}
\hline Telescope & Filter & $\begin{array}{c}\text { Exposure Time } \\
(\mathrm{hr})\end{array}$ & Observation Date \\
\hline \multirow[t]{8}{*}{ KPNO ..................... } & $K_{s}$ & 3.6 & 2004 Feb 5 \\
\hline & $K_{s}$ & 4.3 & 2004 Feb 6 \\
\hline & $K_{s}$ & 5.9 & 2004 Feb 7 \\
\hline & $K_{s}$ & 5.0 & 2004 Feb 8 \\
\hline & $K_{s}$ & 5.2 & 2004 Feb 9 \\
\hline & $K_{s}$ & 3.8 & 2005 Mar 31 \\
\hline & $K_{s}$ & 3.5 & 2005 Apr 1 \\
\hline & $K_{s}$ & 5.6 & 2005 Apr 2 \\
\hline \multirow[t]{11}{*}{ CTIO ..................... } & $K_{s}$ & 0.3 & 2004 Apr 5 \\
\hline & $K_{s}$ & 3.2 & 2004 Apr 6 \\
\hline & $K_{s}$ & 3.8 & 2004 Apr 7 \\
\hline & $K_{s}$ & 3.8 & 2004 Apr 8 \\
\hline & $K_{s}$ & 3.7 & 2004 Apr 9 \\
\hline & $K_{s}$ & 3.2 & 2004 Apr 10 \\
\hline & $K_{s}$ & 2.4 & 2005 Mar 29 \\
\hline & $K_{s}$ & 3.4 & 2005 Mar 30 \\
\hline & $K_{s}$ & 2.8 & 2005 Mar 31 \\
\hline & $K_{s}$ & 2.8 & 2005 Apr 1 \\
\hline & $K_{s}$ & 3.0 & 2005 Apr 2 \\
\hline
\end{tabular}

flat fields, were combined using the same astrometric solution. The image scale for the final stack was set to $0.15^{\prime \prime}$ pixel $^{-1}$.

The $u^{*}$-band images were processed 12 months after the $i^{*}$ images. The reduction was similar, except that a new TERAPIX tool Scamp was used to compute the global astrometric and photometric solutions for the $u^{*}$ images. Finally, a range of quality assessment tests were conducted on the final images, similar to those described in McCracken et al. (2003).

\subsection{CTIO ISPI and KPNO FLAMINGOS}

The FLAMINGOS camera on the KPNO $4 \mathrm{~m}$ telescope (Elston 1998) and the ISPI camera on the CTIO $4 \mathrm{~m}$ telescope (Probst et al. 2003) both provide a field of view slightly larger than $10^{\prime} \times 10^{\prime}$. These cameras contain a single $2 \mathrm{~K} \times 2 \mathrm{~K}$ HAWAII- 2 infrared array detector, which is sensitive between 0.9 and $2.4 \mu \mathrm{m}$. Data from these instruments were combined to obtain a $K_{s}$-band image covering the entire COSMOS field. However, due to weather and the instrument field of view, the depth varies with position. The average KPNO exposure time is $1596 \mathrm{~s}$ in $K_{s}$ band over the field. With CTIO an exposure time of $1436 \mathrm{~s}$ in $K_{s}$ was obtained over the whole field. The variation in depth is recorded in the noise map discussed in $\S 2.1$. A summary of the observing $\log$ for the KPNO/CTIO observations is given in Table 4.

Eighty-one KPNO or CTIO pointings were required to cover the entire COSMOS field. Every position was covered at least four times with KPNO and three times with CTIO. A second grid of 64 pointings offset by half a pointing was taken with CTIO to ensure photometric consistency. The central nine pointings of the COSMOS field were covered with additional passes at the end of the 2004 KPNO run. At each pointing a rotated five-point die-face dither pattern with a $1^{\prime}$ diameter was used. The central position was offset randomly by a few arcseconds between passes to reduce the likelihood of bad pixels falling on the same portion of the sky.

The data were reduced using $\operatorname{IRAF}^{39}$ with a full double-pass reduction algorithm. In the first pass sky flats were produced from averaged, sigma-clipped subsets of 20-30 dark-subtracted images with similar sky levels. A cross-check of different sky flats throughout

\footnotetext{
39 See http://iraf.noao.edu.
} 
the night shows only low-level, large-scale, slowly changing gradients. A global bad pixel mask is generated from a flat used to identify the dead pixels and a pair of dark exposures used to identify hot pixels. The science data were then dark-subtracted and flatfielded. Accurate positional offsets were determined using IRAF's imalign task using multiple well-detected sources identified by SExtractor (Bertin \& Arnouts 1996) that were common to each dithered data set. The images were then stacked using integer pixel offsets with IRAF's imcombine task. These initial stacks of the science data were used to generate relatively deep object masks through SExtractor's CHECKIMAGE_TYPE = OBJECTS output file. These object masks were used to explicitly mask objects when regenerating the sky flats and in the second pass reduction. Supplementary masks for individual images were made to mask out satellites or other bad regions not included in the global bad pixel mask on a frame-by-frame basis.

In the full second-pass reduction algorithm, we individually subtracted the sky from each science frame with 8-10 temporally adjacent images. Each sky-subtracted image was then flat-fielded with the corresponding object-masked sky flat, and any residual variations in the sky removed by subtracting a constant to yield a zero mean sky level. These individual sky-subtracted images were then masked using a combination of the object mask and any supplementary mask to remove the real sources and bad regions in the sky frames and averaged with $\sigma$-clipping to remove cosmic rays. These images were further cleaned of any nonconstant residual gradients as needed by fitting to the fully masked (object + supplementary + global bad pixel masks) background on a lineby-line basis. The dithered data sets were restacked with the same offsets determined in the first pass using the global and supplementary masks. Finally, an initial astrometric solution was determined using the 15-50 stars on each frame from the USNO-A2 catalog. ${ }^{40}$

After flat-fielding and sky subtraction, frames with seeing worse than $1.5^{\prime \prime}$ were removed, and all remaining frames were visually inspected. Any frame with especially high noise, poor tracking, or other defects was removed. Next, every frame was registered to the COSMOS astrometric catalog with a fourth-order, twodimensional polynomial. The polynomial fits were improved by removing mismatched objects in an iterative fashion until the solution converged (typically in four iterations). The resulting scatter between the fit positions and the final astrometry was always less than $0.3^{\prime \prime}$ independent of position. The large astrometric scatter was due to the $0.3^{\prime \prime}$ pixel size of the detector and poor seeing.

Each image was then scaled so that its photometry agreed with the 2MASS point source catalog (Skrutskie et al. 2006). A shift of 1.852 mag was applied to the 2MASS magnitudes to convert them to the AB system. The $2 \mathrm{MASS}, \mathrm{CTIO}$, and KPNO $K_{s}$ filters are sufficiently similar that no color terms are needed to convert between these filters. After photometric calibration, the scatter between overlapping exposures was measured to remove any position-dependent photometric shifts.

Finally, all data were smoothed with a Gaussian kernel to a $1.5^{\prime \prime}$ FWHM and combined with SWarp to produce images on the COSMOS grid with $0.15^{\prime \prime}$ pixel $^{-1}$. The rms noise maps, derived from the image noise and flat fields, were also combined using the same astrometric solution. An original PSF image was not produced for the $K_{s}$-band data because the PSF variations were too large.

\subsection{Sloan Digital Sky Survey}

SDSS DR2 images (Abazajian et al. 2004) were used to obtain photometry for objects saturated in the Subaru and CFHT data.

40 VizieR Online Data Catalog, 1252 (D. B. A. Monet et al., 1998)
Objects as bright as 10 mag have good photometry in the SDSS images. DR2 was used because later SDSS data releases contained no new data or calibration for the COSMOS field.

To facilitate photometric measurements, a mosaic of the SDSS data was created on the same grid as the other COSMOS data. The data were downloaded and calibrated with the "best" photometric calibration as outlined on the SDSS DR2 Web site. ${ }^{41}$ A median sky level was then subtracted and a catalog generated for each image. The objects in each image were then matched to the COSMOS astrometric catalog using a third-order, two-dimensional polynomial with the IMCAT fitgeometry routine.

The PSF of the SDSS data was homogenized by smoothing all images to the same FWHM with a Gaussian kernel. However, systematic effects on the order of 5\% remained between SDSS "stripes" due to non-Gaussian wings of the SDSS PSF. This is discussed further in $\S 3$. The COSMOS archive also contains a second combination with the non-PSF-homogenized images.

After PSF homogenization, images were resampled with a linear interpolation using the IMCAT warpimage routine. All images in each band were then combined with the IMCAT combineimages routine using a weighted average. An rms noise map was also generated during the image combination process. The image scale for the final stack was set to $0.15^{\prime \prime}$ pixel $^{-1}$.

\section{POINT-SPREAD FUNCTION MATCHING}

A consistent PSF within each band and between bands is essential for high-quality photometry. Ideally, all bands would have an identical PSF, but achieving a homogeneous PSF for a data set as diverse as COSMOS is extremely difficult due to the nonGaussian portion of most PSFs. To ensure a consistent PSF across the field of view and between bands we adopted a two-step process. First we homogenized the PSF within each band during the data reduction, then we matched the homogenized PSFs to the band with the worst image quality.

Within each band, we adopted a Gaussian kernel to homogenize the PSF between exposures. This works well if the seeing variations are small, there are many images at the same position to average out the PSF, and the photometric apertures are much larger than the seeing size. These assumptions, however, break down for large seeing variations and small numbers of images. In the COSMOS data the effect of non-Gaussian PSF components is negligible $(\leq 0.01 \mathrm{mag})$ for all but the SDSS data when using the PSF-matched 3 " aperture photometry.

The SDSS data consist of a single exposure at each position with seeing variations as large as $1^{\prime \prime}$ between the two nights which cover the COSMOS field (see $\S \S 2$ and 2.5). These data are collected in five parallel strips in right ascension with a detector-wide gap in declination between strips. Two passes are required to completely cover an area of the sky. Figure 7 shows the offset between the SDSS $r$-band and the Subaru $r^{+}$-band photometry measured in a $3^{\prime \prime}$ aperture as a function of right ascension and declination. An offset of $0.06 \mathrm{mag}$ is clearly seen in declination between the two SDSS passes due to the imperfect PSF matching. This offset is not visible if total magnitudes, which correct for seeing variation, are compared (see $\S 5.1$ ). The offsets are similar in all SDSS bands, so colors between SDSS bands are not significantly impacted.

For stellar photometry measured with the Subaru data in small $\left(\simeq 1^{\prime \prime}\right)$ apertures, a magnitude-dependent aperture correction is required between 18 and $21 \mathrm{mag}$. The correction is due to differences in seeing between the short- and long-exposure data taken with Suprime-Cam. The short-exposure data were typically taken at higher air mass, and hence worse seeing, than the long-exposure

41 See http://www.sdss.org/dr2/. 

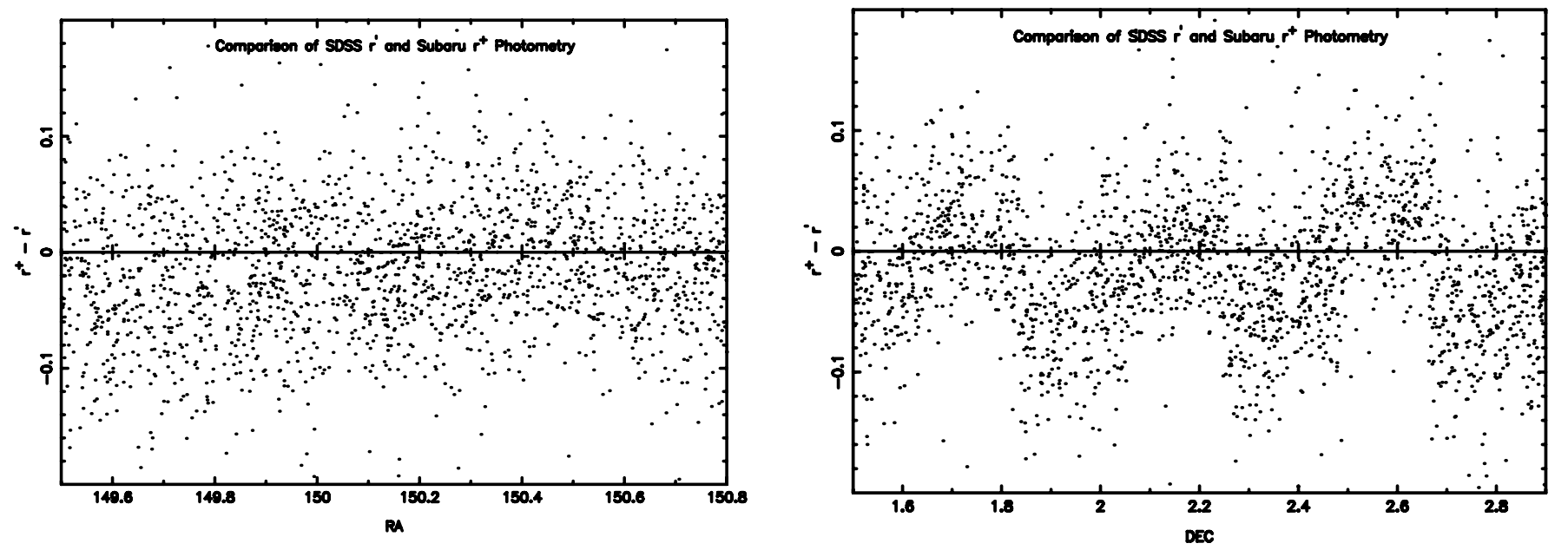

FIG. 7.-Photometric offsets for stars between the SDSS $r$ and Subaru $r^{+}$data as a function of right ascension (left $)$and declination $(r i g h t)$. Notice there are no systematics in right ascension, but there is a steplike pattern in declination which corresponds to two different SDSS runs. The step pattern is due to imperfect PSF matching.

data. In addition, fewer exposures were taken with shorter than longer exposure times. As a result, the long-exposure data are smoothed with a much larger kernel than the short-exposure data, resulting in better PSF matching at fainter magnitudes. Corrections for this effect are given for several bands in Robin et al. (2007), which uses a different photometric catalog from the one presented here.

To avoid these PSF-matching problems in the multiband catalog, the PSF matching was optimized for a $3^{\prime \prime}$ aperture. This was achieved by convolving each PSF-homogenized image with a Gaussian kernel that produced the same flux ratio between a $3^{\prime \prime}$ and $10^{\prime \prime}$ aperture for a point source. This method is superior to simply matching the FWHM since it accounts for the non-Gaussian parts of the PSF. However, with this method, only 3 " apertures are free from systematic effects.

We selected the kernel for each image by identifying point sources in the ACS images, then using these to construct a median PSF for each of the PSF-homogenized images. A Gaussian kernel was then selected which yielded the same flux ratio between a $3^{\prime \prime}$ and $10^{\prime \prime}$ aperture as the band with the smallest ratio (the $K_{s}$ image). These smoothing kernels are listed in Table 5.

TABLE 5

PSF Properties and Smoothing Kernels Used for Photometry

\begin{tabular}{|c|c|c|c|}
\hline Filter & $\begin{array}{l}\text { PSF FWHM } \\
\text { (arcsec) }\end{array}$ & $\begin{array}{l}\text { Fraction of Flux } \\
\text { in } 3^{\prime \prime} \text { Aperture }\end{array}$ & $\begin{array}{l}\text { Smoothing Kernel } \sigma^{\mathrm{a}} \\
\text { (arcsec) }\end{array}$ \\
\hline 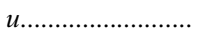 & 1.97 & 0.716 & 0.000 \\
\hline$u^{*} \ldots \ldots \ldots \ldots \ldots \ldots$ & 0.90 & 0.919 & 0.662 \\
\hline$B_{\mathrm{J}}$ & 0.95 & 0.942 & 0.699 \\
\hline$g$ & 1.97 & 0.725 & 0.000 \\
\hline$g^{+} \ldots \ldots \ldots \ldots \ldots \ldots \ldots \ldots$ & 1.58 & 0.795 & 0.240 \\
\hline$V_{\mathrm{J}} \ldots \ldots \ldots \ldots \ldots \ldots$ & 1.33 & 0.874 & 0.521 \\
\hline$r^{+} \ldots \ldots \ldots \ldots \ldots \ldots \ldots \ldots$ & 1.05 & 0.914 & 0.639 \\
\hline$r$ & 1.97 & 0.708 & 0.000 \\
\hline$i$ & 1.97 & 0.709 & 0.000 \\
\hline$i^{+} \ldots \ldots \ldots \ldots \ldots \ldots \ldots$ & 0.95 & 0.914 & 0.611 \\
\hline$i^{*}$ & 0.95 & 0.891 & 0.620 \\
\hline NB816 .................. & 1.51 & 0.851 & 0.463 \\
\hline F814W.................. & 0.07 & 0.979 & 0.785 \\
\hline$z$ & 1.97 & 0.701 & 0.000 \\
\hline$z^{+}$ & 1.15 & 0.866 & 0.585 \\
\hline$K_{s} \ldots \ldots \ldots \ldots \ldots \ldots \ldots \ldots \ldots \ldots \ldots \ldots$ & 1.50 & 0.759 & 0.000 \\
\hline
\end{tabular}

${ }^{\mathrm{a}} \mathrm{FWHM}=2 \sqrt{2 \ln (2)} \sigma$.
With the exception of the SDSS images, the worst seeing image was the $K_{S}$ band, which contained only $76 \%$ of the flux in a $3^{\prime \prime}$ aperture. To account for the fact that the SDSS images have seeing worse than the $K_{s}$ image, an aperture correction of -0.06 mag was applied to the photometry from these bands.

\section{THE MULTIBAND CATALOG}

The COSMOS multiband catalog is derived from a combination of the CFHT $i^{*}$ and Subaru $i^{+}$original-PSF images. The CFHT $i^{*}$-band image alone is too shallow, while compact objects in the Subaru $i^{+}$image saturate at $21 \mathrm{mag}$. Therefore, a combination of the two gives the largest possible dynamic range for a detection image. The resulting catalog is well matched in wavelength and depth to the HST ACS catalog (Leauthaud et al. 2007) and optimally deblends the ground-based photometry. The catalog is also optimal for many science goals which require photometric redshifts.

A $\chi^{2}$ image constructed from multiple bands was also tried as a detection image but then rejected. The main advantage of a $\chi^{2}$ image is panchromatic completeness. However, only a small number of faint objects with very extreme colors are detected in a $\chi^{2}$ image but not in the $i^{+}$-band image due to the depth and quality of the $i^{+}$-band data. As a result of their faint magnitudes and extreme colors, these objects will have poor photometric redshifts, so detecting them is of limited use. Furthermore, the scientific drivers for a $\chi^{2}$ image strongly favor including the $K_{s}$ band, which has $1.5^{\prime \prime}$ seeing. This poor seeing significantly reduces the ability to split close pairs of objects, so the benefits of a $\chi^{2}$ image are outweighed by the loss in resolution.

The catalog was generated with SExtractor (Bertin \& Arnouts 1996 ) run in dual-image mode on each of the 144 image tiles (see $\S 2.1$ ). Only objects in the central $10^{\prime} \times 10^{\prime}$ of each tile were recorded to avoid duplicate detections in the overlapping regions. The combined $i^{+}$- and $i^{*}$-band image was used as the detection image for all bands. Absolute rms maps were used as weight maps for both the detection and measurement images. ${ }^{42}$ Measurements were done on the PSF-homogenized images, further smoothed to PSF-match all bands (see $\S 3$ ).

\footnotetext{
42 The default SExtractor settings assume variations in noise reflect variations in gain across the image. However, in this case both the data and rms images are in absolute flux units, so the gain is 1 everywhere by definition. The SExtractor parameter WEIGHT_GAIN must be set to $\mathrm{N}$ to avoid the default behavior. Failure to set WEIGHT_GAIN $=\mathrm{N}$ will result in incorrect error estimates.
} 
TABLE 6

SExtractor Parameters

\begin{tabular}{|c|c|c|}
\hline Parameter & Setting & Comment \\
\hline PARAMETERS_NAME. & cosmos.param & Fields to be included in output catalog \\
\hline 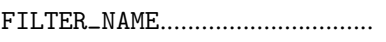 & gauss_2.5_5x5.conv & Filter for detection image \\
\hline STARNNW_NAME.......................... & default.nnw & Neural-network_weight table filename \\
\hline CATALOG_NAME......................... & STDOUT & Output to pipe instead of file \\
\hline CATALOG_TYPE........................... & ASCII & Output type \\
\hline DETECT_TYPE............................. & CCD & Detector type \\
\hline DETECT_MINAREA ....................... & 3 & Minimum number of pixels above threshold \\
\hline DETECT_THRESH ......................... & 0.6 & Detection threshold in $\sigma$ \\
\hline ANALYSIS_THRESH ..................... & 0.6 & Limit for isophotal analysis $\sigma$ \\
\hline 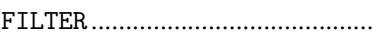 & $Y$ & Use filtering \\
\hline DEBLEND_NTHRESH .................... & 64 & Number of deblending subthresholds \\
\hline DEBLEND_MINCONT ...................... & 0.0 & Minimum contrast parameter for deblending \\
\hline 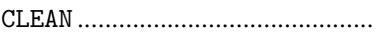 & $Y$ & Clean spurious detections \\
\hline CLEAN_PARAM............................. & 1 & Cleaning efficiency \\
\hline 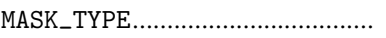 & CORRECT & Correct flux for blended objects \\
\hline PHOT_APERTURES ...................... & $6.7,13.3,20,26.7,33.3,66.7$ & MAG_APER aperture diameter(s) in pixels \\
\hline PHOT_AUTOPARAMS ...................... & $2.5,3.5$ & MAG_AUTO parameters: $\langle$ Kron_fact $\rangle,\langle$ min_radius $\rangle$ \\
\hline PHOT_FLUXFRAC ......................... & $0.2,0.5,0.8,0.9$ & Define $n$ light radii \\
\hline PHOT_AUTOAPERS ....................... & $20.0,20.0$ & MAG_AUTO minimum apertures: estimation, photometry \\
\hline SATUR_LEVEL............................. & 200000 & Level of saturation \\
\hline MAG_ZEROPOINT ......................... & 31.4 & Magnitude zero point \\
\hline 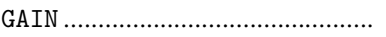 & 1 & Gain is 1 for absolute rms map \\
\hline PIXEL_SCALE............................. & 0.1500 & Size of pixel in arcseconds \\
\hline SEEING_FWHM............................. & 0.95 & Stellar FWHM in arcseconds \\
\hline 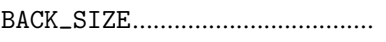 & 256 & Background mesh in pixels \\
\hline BACK_FILTERSIZE ..................... & 5 & Background filter \\
\hline BACKPHOTO_TYPE ...................... & LOCAL & Photometry background subtraction type \\
\hline BACKPHOTO_THICK ..................... & 120 & Thickness of the background LOCAL annulus \\
\hline WEIGHT_GAIN............................ & $\mathrm{N}$ & Gain does not vary with changes in rms noise \\
\hline WEIGHT_TYPE........................... & MAP_RMS & Set weight image type \\
\hline MEMORY_PIXSTACK ..................... & 5000000 & Number of pixels in stack \\
\hline MEMORY_BUFSIZE ..................... & 4096 & Number of lines in buffer \\
\hline MEMORY_OBJSTACK ..................... & 60000 & Size of the buffer containing objects \\
\hline VERBOSE_TYPE............................. & QUIET & \\
\hline INTERP_MAXXLAG ....................... & 3 & Number of bad pixels to interpolate over in $X$ \\
\hline INTERP_MAXYLAG ...................... & 3 & Number of bad pixels to interpolate over in $Y$ \\
\hline INTERP_TYPE........................... & ALL & Type of interpolation \\
\hline
\end{tabular}

The number of contiguous pixels, detection thresholds, deblending parameters, and smoothing kernels were adjusted to maximize completeness when visually compared to the ACS and $\chi^{2}$ image. Setting these parameters aggressively results in false detections around bright stars and residual image defects. However, these false detections can be removed by requiring $5 \sigma$ measurements in the detection band, a reasonable FWHM, and a defect mask for the detection image. The SExtractor parameters are given in Table 6, while the software used to generate these catalogs is available at the COSMOS Web site. ${ }^{43}$

Aperture photometry with a $3^{\prime \prime}$ diameter is measured for each band. This provides the best possible color measurements by minimizing the effects of PSF variation from band to band (see $\S 3$ ). Total magnitudes are suboptimal for color measurements because a correction factor must be estimated separately for each band, increasing the error. Furthermore, SExtractor estimates this correction only on the detection image, so estimates of total magnitude are only accurate if the image quality of the detection and measurement image are identical.

In contrast, properly PSF-matched images have identical aperture corrections in all bands, so a single estimate of the total

43 See http://cosmos.astro.caltech.edu. magnitude can be used for all bands. We estimate the correction to total magnitude using the offset between the total (MAG_AUTO) and the $3^{\prime \prime}$ aperture magnitude in the detection image. This difference can be added to any aperture magnitude to yield a total magnitude.

The median $5 \sigma$ depths for the catalog including PSF matching, deblending, background subtraction, and photon noise are given in Table 7. These numbers are for total magnitudes and should be used when choosing signal-to-noise ratio cuts and magnitude limits for the COSMOS catalog. An estimate of the rms variation in the $5 \sigma$ limiting magnitude, along with the upper and lower quartiles of the $5 \sigma$ limiting magnitude, is also given.

The general release catalog is cut at a total $i^{+}$magnitude of 25 and only includes the $2 \mathrm{deg}^{2}$ with uniform multiband coverage. At this magnitude limit and in this area photometric redshifts are reliable, the catalog is complete, and spurious sources are minimal. At fainter magnitudes the catalog begins to be incomplete and have more spurious detections, and photometric redshifts begin to behave poorly. A full catalog of all detections is available on request. However, the full catalog should be used with caution. In particular, the completeness and the number of spurious sources will vary as a function of position due to differences in the rms background noise. 
TABLE 7

Depth of $i$-Band Catalog Photometry

\begin{tabular}{|c|c|c|c|c|}
\hline Filter & $\begin{array}{l}5 \sigma \text { Depth in } \\
3^{\prime \prime} \text { Aperture }\end{array}$ & $\begin{array}{l}\text { rms Range } \\
\text { of Depth }\end{array}$ & $\begin{array}{c}\text { Upper } \\
\text { Quartile }^{\mathrm{a}}\end{array}$ & $\begin{array}{c}\text { Lower } \\
\text { Quartile }^{\mathrm{b}}\end{array}$ \\
\hline 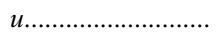 & 23.2 & 0.3 & 22.9 & 23.4 \\
\hline 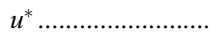 & 26.5 & 0.2 & 26.3 & 26.6 \\
\hline$B_{\mathrm{J}} \ldots \ldots \ldots \ldots \ldots \ldots \ldots \ldots \ldots \ldots \ldots \ldots$ & 26.6 & 0.1 & 26.6 & 26.7 \\
\hline$g \ldots \ldots \ldots \ldots \ldots \ldots \ldots$ & 23.9 & 0.2 & 23.9 & 23.9 \\
\hline$g^{+} \ldots \ldots \ldots \ldots \ldots \ldots \ldots \ldots$ & 26.5 & 0.2 & 26.5 & 26.6 \\
\hline$V_{\mathrm{J}} \ldots \ldots \ldots \ldots \ldots \ldots \ldots \ldots \ldots \ldots$ & 26.5 & 0.2 & 26.4 & 26.5 \\
\hline 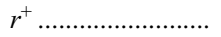 & 26.6 & 0.2 & 26.5 & 26.6 \\
\hline 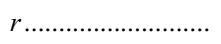 & 23.6 & 0.3 & 23.4 & 23.8 \\
\hline$i$ & 22.9 & 0.3 & 22.8 & 23.2 \\
\hline$i^{+} \ldots \ldots \ldots \ldots \ldots \ldots \ldots \ldots$ & 26.1 & 0.2 & 26.0 & 26.2 \\
\hline$i^{*} \ldots \ldots \ldots \ldots \ldots \ldots \ldots \ldots$ & 23.5 & 0.3 & 23.3 & 23.7 \\
\hline NB816 .................... & 25.5 & 0.2 & 25.4 & 25.6 \\
\hline F814W ..................... & 25.3 & 0.1 & 25.3 & 25.4 \\
\hline 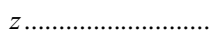 & 21.5 & 0.3 & 21.3 & 21.7 \\
\hline$z^{+} \ldots \ldots \ldots \ldots \ldots \ldots \ldots \ldots \ldots \ldots \ldots \ldots$ & 25.1 & 0.2 & 25.1 & 25.2 \\
\hline$K_{s} \ldots \ldots \ldots \ldots \ldots \ldots \ldots \ldots \ldots \ldots \ldots \ldots \ldots$ & 21.2 & 0.3 & 21.1 & 21.3 \\
\hline
\end{tabular}

${ }^{a}$ Twenty-five percent of objects with $5 \sigma$ measurements are brighter than this magnitude.

b Twenty-five percent of objects with $5 \sigma$ measurements are fainter than this magnitude.

\subsection{Catalog Contents}

The multiband catalog contains PSF-matched 3 " aperture photometry and errors for all Subaru and CFHT bands along with the $\mathrm{KPNO}+\mathrm{CTIO} K_{s}$-band and HST F814W-band data. Non-PSFmatched photometry is also included for the SDSS bands. Objects with no detection are assigned a magnitude of 99 and an error indicating the $1 \sigma$ limiting magnitude expected for that band. Objects with no measurement due to lack of coverage, saturation, or other defects are assigned a magnitude and error of -99 .

The catalog photometry uses the photometric zero points determined by the standard stars, which are known to have systematic offsets on the order of $0.05 \mathrm{mag}$ and up to $0.2 \mathrm{mag}$ in the $B_{\mathrm{J}}$ band. We strongly suggest applying the zero-point corrections given in Table 13 and discussed in $\S 5.3$ to get the best possible photometry.

The total (MAG_AUTO) magnitude and the FWHM, which is measured from either the PSF-matched Subaru $i^{+}$-band or the PSFmatched CFHT $i^{*}$-band image (if the Subaru data are missing or saturated), are included in the catalog along with a flag indicating which image was used. As discussed in the previous section, since the photometry is PSF-matched the difference between the total and aperture magnitudes in the combined $i$-band data provides an aperture correction for each object. This aperture correction is also included in the catalog. Applying this aperture correction to any band will provide a total magnitude in that band.

A flag indicating that the object may be a star instead of a galaxy is also included; however, this flag is a qualitative assessment, and science relying on accurate star/galaxy separation should perform a more detailed analysis. Quantitative indicators such as the SExtractor CLASS_STAR parameter are ineffective at separating stars and galaxies because the FWHM varies as a function of magnitude on the detection image. Furthermore, we could not use the HST ACS data to separate point sources because they only cover a fraction of the deep ground-based data. To overcome this limitation we manually defined regions on a plot of detection image FWHM versus magnitude, which contained the majority of ACS point sources (objects with CLASS_STAR $>0.9$ in the ACS catalog; Leauthaud et al. 2007). Objects falling in this region were then flagged as stars in the ground-based catalog.

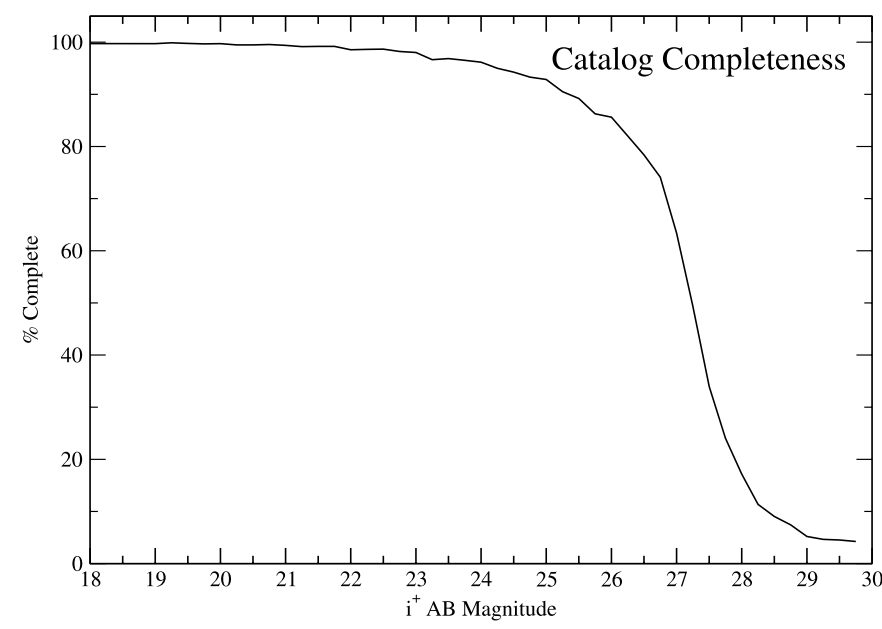

FIG. 8.-Estimated completeness of the $i^{+}$-band-detected catalog as a function of magnitude using the method described in McCracken et al. (2003). This method randomly places objects with a representative range of morphologies in the field and attempts to recover them. The estimate includes the effects of blending and detection completeness.

Flags marking masked regions in the Subaru $B_{\mathrm{J}}, V_{\mathrm{J}}, i^{+}$, and $z^{+}$ images are included in the catalog. These masks were generated by visually inspecting images, using the SDSS magnitudes as a guide to flag bright stars and estimate the size of masks. The flag value is the area in square arcseconds of the photometry aperture which falls inside a masked region.

Finally, a flag indicating heavily deblended objects is included in the catalog. The default SExtractor deblending settings fail to find objects in areas around bright objects due to the high dynamic range of the $i^{+}$-band detection image. Unfortunately, deblending aggressively enough to find faint objects around bright ones results in numerous false detections near the bright objects. To mitigate this problem, objects not detected by the default SExtractor deblending parameters are flagged.

\subsection{Catalog Usage Guide}

Objects with all mask and deblending flags set to 0 have the most reliable photometry. However, this removes a significant fraction of the survey area, and some flags can be safely ignored under certain circumstances.

When cross-correlating with multiwavelength detections, the deblending and star flags can often be safely ignored. However, for clustering analysis based on the optical catalog the deblending flag is very important, especially at faint magnitudes (see McCracken et al. 2007). Furthermore, the photometry of faint objects deblended from extended nearby galaxies is suspect due to color gradients in the nearby object.

The photometry masks are specific to the band in which they were measured. But since the same instrument (Suprime-Cam) was used for most of the photometry, they can be safely extended to adjacent bands (for instance, a combination of the $B_{\mathrm{J}}$ and $V_{\mathrm{J}}$ mask is appropriate for the $g^{+}$photometry). Photometric redshifts are affected by masked photometry in a nonlinear fashion, so all photometry masks must be applied to obtain a clean photometric redshift sample. However, in specific redshift ranges some bands are not as important, so the masking could be relaxed if a spectroscopic control sample is available.

\subsection{Completeness and Confusion}

For deep surveys the ability to detect objects (completeness) and separate superimposed objects (confusion) are often more 
TABLE 8

Color Conversion between SDSS and COSMOS Photometric Systems

\begin{tabular}{|c|c|c|}
\hline COSMOS Filter & Valid for & Conversion Equation $^{\mathrm{a}}$ \\
\hline & $-1<(g-r)<1$ & $B_{\mathrm{J}}=g+0.240(g-r)+0.029$ \\
\hline$g^{+} \ldots \ldots$ & $-1<(g-r)<1$ & $056(g-r)-0.006$ \\
\hline & $-1<(g-r)<1$ & $17(g-r)-0.021$ \\
\hline & $-1<(g-r)<1$ & $r^{+}=r-0.037(g-r)+0.003$ \\
\hline & $-1<(r-i)<1$ & $i^{+}=i-0.106(r-i)+0.007$ \\
\hline$z^{+} \ldots \ldots \ldots \ldots \ldots \ldots \ldots \ldots \ldots$ & $-1<(i-z)<0.8$ & $z^{+}=z-0.110(i-z)+0.008$ \\
\hline
\end{tabular}

a A comparison between SDSS $u$ and CFHT $u^{*}$ is not possible because there is no simple linear relationship between these filters.

important sources of measurement error than the photon noise. These quantities are more difficult to quantify than the formal noise given in Table 2 because they are sensitive to both the data quality and the software used.

The image quality, or seeing, has a much larger impact on completeness and confusion than the measurement error. For a fixed aperture the measurement error increases linearly with FWHM, while the peak flux decreases as $\mathrm{FWHM}^{-1}$, and the confusion increases as $\mathrm{FWHM}^{2}$. So a factor of 2 difference in seeing corresponds to a 0.3 mag reduction in measurement sensitivity, but a 0.75 mag reduction in peak surface brightness and a factor of 4 increase in blending. As a result, it is often possible to achieve much greater flux measurement depth than detection depth. SExtractor uses a peak-finding algorithm which is especially sensitive to image quality and software settings. The threshold, pixel area, and smoothing kernel settings have a large impact on completeness, while the deblending parameters impact confusion. These settings are often a compromise between detection depth and the number of spurious detections.

For the COSMOS catalog we chose parameters which maximized detection depth but also produced spurious detections near the detection limit. These spurious objects can be removed with object masks and cuts in magnitude and FWHM. A similar method to ours is employed by the CFHT-LS survey. However, other groups such as the Subaru $X M M$ Deep Survey have preferred more conservative settings, which minimize spurious sources at the expense of detection sensitivity. These more conservative numbers are given for all Subaru bands in Taniguchi et al. (2007).

To quantify completeness, simulated objects with a representative range of morphologies and magnitudes are inserted into the image. SExtractor is then run on the image, and the fraction of recovered objects at each magnitude is measured. No attempt is made to avoid existing objects, so the effects of confusion are included in the completeness calculation. This is identical to the method described in McCracken et al. (2003). Figure 8 shows the results of this simulation for the combined $i^{+}$and $i^{*}$ detection im- age. The catalog is $91 \%$ complete at $i^{+}=25.0,87 \%$ complete at $i^{+}=26.0$, and $50 \%$ complete at $i^{+}=27.4$.

\section{DISCUSSION}

\subsection{Consistency of Photometry}

The COSMOS survey was designed to probe the interplay of large-scale structure and galaxy evolution. High-quality photometric redshifts are essential for these studies. Uncertainties greater than $2 \%$ in redshift will begin to wash out large-scale structure (Scoville et al. 2007a), so systematic variations in photo- $z$ values need to be less than $1 \%$. Since a $1 \%$ error in photometry typically leads to a $1 \%$ error in photo-z, this places extremely high requirements on the input photometry quality.

The Canada-France-Hawaii Telescope Legacy Survey (CFHTLS) and the SDSS have independently covered the COSMOS field, allowing for a quantitative estimate of our photometric consistency. The Subaru $i^{+}$, CFHT $i^{*}$, and HST ACS F814W data have similar bandpasses but were collected on different instruments and reduced by different teams using different software, allowing for an internal check on photometric consistency.

In our comparisons the best effort at estimating total magnitudes from each survey is used to minimize the effects of aperture size. The specific magnitudes used were SExtractor MAG_AUTO values for CFHT-LS, Petrosian magnitudes for SDSS, and aperture magnitudes corrected to total (as described in $\S 4$ ) for COSMOS. The CFHT-LS and SDSS photometry were then converted to the COSMOS filter system using the conversions given in Tables 8 and 9 and discussed further in H. Aussel et al. (2007, in preparation). Finally, the catalogs were merged by position with COSMOS, allowing for a $1^{\prime \prime}$ offset in position.

Figure 9 shows a comparison between the COSMOS data and those from the CFHT-LS and SDSS, as well as between the Subaru $i^{+}$, CFHT $i^{*}$, and HST ACS F814W bands for objects brighter than $25 \mathrm{mag}$ in the COSMOS ground-based data and magnitude errors smaller than $0.21(5 \sigma)$ in the comparison data. A comparison between SDSS $u$ and CFHT $u^{*}$ is not possible because there is no simple linear relationship between these filters. No systematic effects as a function of position are measurable within the photometric uncertainty for the $1^{\prime}$ bins used for CFHT-LS and $2^{\prime}$ bins used for SDSS. Smoothing on the size scale of a Suprime-Cam pointing yields a typical $\mathrm{rms}$ variation of 0.01 mag across the field between COSMOS, the CFHT-LS, and SDSS (see Table 10), while a comparison between the COSMOS CFHT $i^{*}$ and Subaru $i^{+}$gives 0.007 mag of scatter $(1 \sigma)$, and the ACS F814W data and Subaru $i^{+}$give a dispersion of 0.003 mag.

Figure 9 also compares the CTIO and KPNO $K_{s}$-band photometry to those from 2MASS. Objects with total magnitudes of $i^{+}>16$ and at least a $5 \sigma$ detection in $K_{s}$ were used in the comparison. No systematic trend is measurable with position, and the

TABLE 9

Color Conversion Between CFHT-LS and COSMOS Photometric Systems

\begin{tabular}{|c|c|c|}
\hline COSMOS Filter & Valid for & Conversion Equation \\
\hline$u^{*} \ldots \ldots \ldots \ldots \ldots$ & All & Same filter \\
\hline 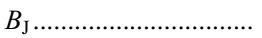 & $-0.2<\left(g^{*}-r^{*}\right)<0.6$ & $B_{\mathrm{J}}=g^{*}+0.432\left(g^{*}-r^{*}\right)+0.047$ \\
\hline 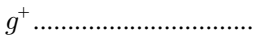 & $-1.0<\left(g^{*}-r^{*}\right)<0.6$ & $g^{+}=g^{*}+0.094\left(g^{*}-r^{*}\right)+0.008$ \\
\hline 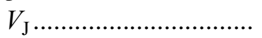 & $-0.2<\left(g^{*}-r^{*}\right)<0.6$ & $V_{\mathrm{J}}=g^{*}-0.545\left(g^{*}-r^{*}\right)-0.016$ \\
\hline 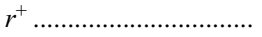 & $-1.0<\left(g^{*}-r^{*}\right)<0.9$ & $r^{+}=r^{*}-0.021\left(g^{*}-r^{*}\right)+0.001$ \\
\hline 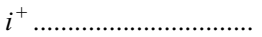 & $-1.0<\left(g^{*}-r^{*}\right)<0.8$ & $i^{+}=i^{*}-0.020\left(g^{*}-r^{*}\right)+0.005$ \\
\hline F814W........................... & $0.5<\left(i^{*}-z^{*}\right)<0.0$ & $\mathrm{~F} 814 \mathrm{~W}=z^{*}+0.632\left(i^{*}-z^{*}\right)-0.116\left(i^{*}-z^{*}\right)^{2}-0.001$ \\
\hline 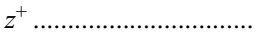 & $-1.0<\left(i^{*}-z^{*}\right)<0.8$ & $z^{+}=z^{*}-0.128\left(i^{*}-z^{*}\right)-0.004$ \\
\hline
\end{tabular}




\section{CFHT-LS SDSS}
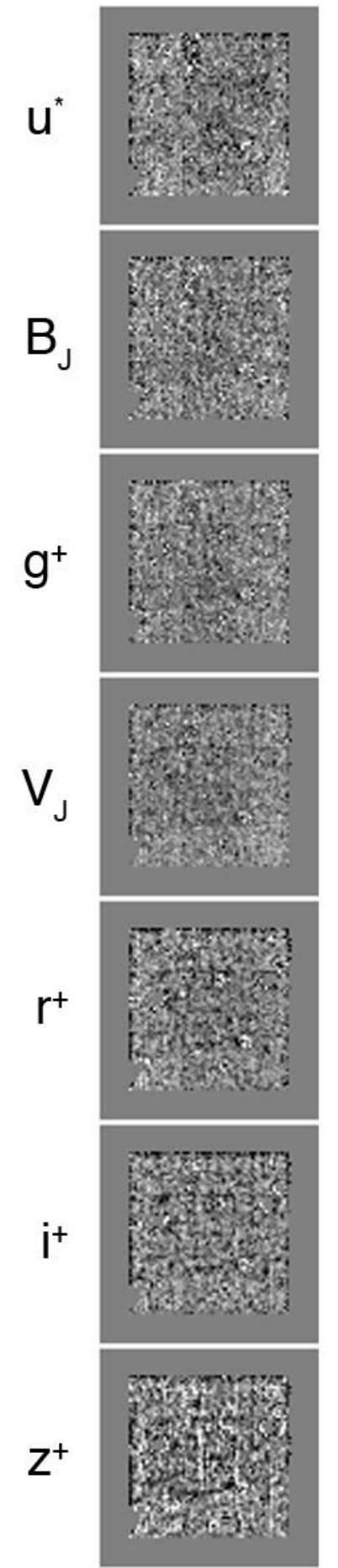
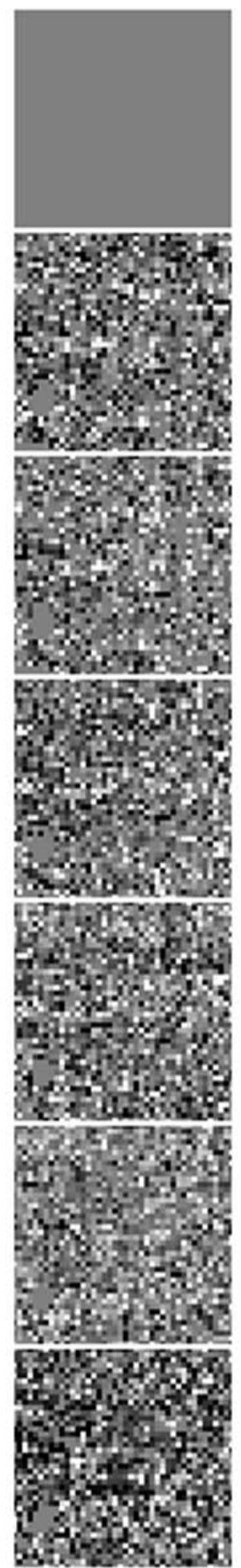

\section{Subaru i+}

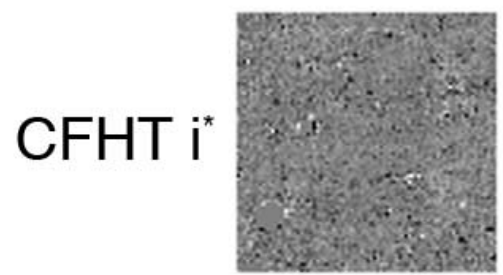

F814W

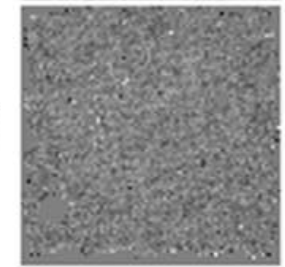

\section{MASS Ks}
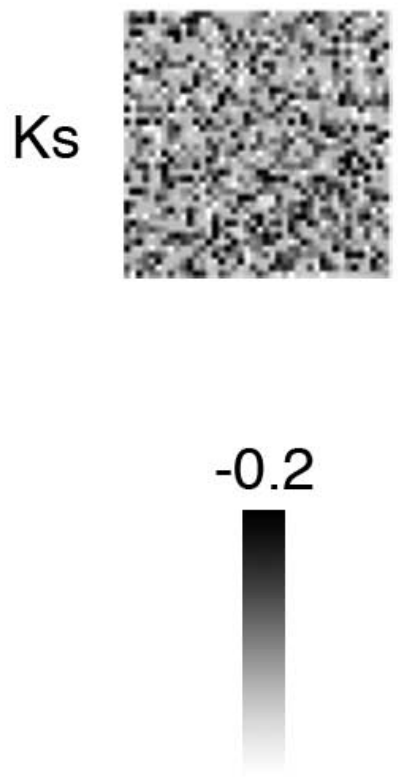

$+0.2$

FIG. 9.-Gray-scale images showing offsets between photometry measured by the CFHT-LS and SDSS surveys and COSMOS (left), between Subaru $i^{+}$, CFHT $i^{*}$, and ACS F814W bands within the COSMOS survey (top right), and between 2MASS and the COSMOS $K_{s}$ band (middle right). The images have $1^{\prime}$ pixels for all surveys but SDSS and 2MASS, which have $2^{\prime}$ pixels. The CFHT-LS D2 field only covers the central $1 \mathrm{deg}^{2}$ of COSMOS, resulting in a blank area. A comparison between SDSS $u$ and CFHT $u^{*}$ is not possible because there is no simple linear relationship between these filters. The scale is linear in magnitude from black $(-0.2 \mathrm{mag})$ to white $(+0.2 \mathrm{mag})$. No variation with position is measurable within the measurement errors. 
TABLE 10

rms Variation in the Photometric Zero Point as a Function of Position between Surveys

\begin{tabular}{|c|c|c|}
\hline COSMOS Filter & CFHT-LS & SDSS \\
\hline$u^{*}$ & 0.014 & $\ldots^{\mathrm{a}}$ \\
\hline 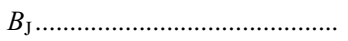 & 0.009 & 0.006 \\
\hline 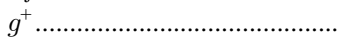 & 0.010 & 0.013 \\
\hline 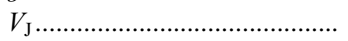 & 0.010 & 0.020 \\
\hline$r^{+}$ & 0.013 & 0.009 \\
\hline$i^{+}$ & 0.007 & 0.012 \\
\hline 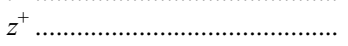 & 0.010 & 0.017 \\
\hline
\end{tabular}

a A comparison between $\operatorname{SDSS} u$ and CFHT $u^{*}$ is not possible because there is no simple linear relationship between these filters.

rms variation between COSMOS and 2MASS is 0.02 mag on the scale of a CTIO/KPNO pointing. At magnitudes brighter than $i^{+}<16$ the detection image is saturated, resulting in an incorrect aperture correction even though the $K_{s}$-band photometry is unsaturated. This is manifested as an apparent systematic trend with magnitude between the COSMOS catalog and 2MASS photometry. However, no trend is visible for objects fainter than $i^{+}>16$ and with measurements made directly on the $K_{s}$-band image.

The ACS photometry has the best relative calibration of all the data sets due to the lack of atmospheric absorption. So the excellent agreement between the ACS and Subaru $i^{+}$photometry as a function of position indicates that the variations seen between COSMOS and the CFHT-LS and SDSS are largely due to flatfielding or sky-subtraction errors in those other surveys. Nevertheless, our photometry appears to be constant across the COSMOS field to better than $1 \%$, meeting the science requirement for largescale structure studies.

\subsection{Galactic Extinction Correction}

The median Galactic extinction in the COSMOS field is $e(B-V)=0.0195 \pm 0.006$, which corresponds to an extinction of $0.10 \pm 0.03 \mathrm{mag}$ in the $u^{*}$ band. The estimated Galactic extinction from Schlegel et al. (1998) is provided for each object in the COSMOS catalog. A photometric correction for each band can be determined from the Galactic extinction multiplied by a

TABLE 11

Galactic Extinction Corrections

\begin{tabular}{|c|c|c|}
\hline Filter & $k_{\lambda}{ }^{\mathrm{a}}$ & Median $A_{\lambda}$ \\
\hline и. & 4.996724 & 0.097436 \\
\hline$u^{*}$ & 4.690237 & 0.091460 \\
\hline$B_{\mathrm{J}} \ldots \ldots \ldots \ldots \ldots \ldots \ldots$ & 4.038605 & 0.078753 \\
\hline 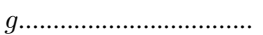 & 3.791856 & 0.073941 \\
\hline$g^{+} \ldots \ldots \ldots \ldots \ldots \ldots \ldots \ldots \ldots$ & 3.738239 & 0.072896 \\
\hline$V_{\mathrm{J}}$ & 3.147140 & 0.061369 \\
\hline 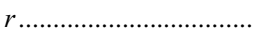 & 2.649158 & 0.051659 \\
\hline$r^{+} \ldots \ldots \ldots \ldots \ldots \ldots \ldots \ldots \ldots \ldots$ & 2.586050 & 0.050428 \\
\hline$i$ & 1.989881 & 0.038803 \\
\hline$i^{+}$ & 1.922693 & 0.037493 \\
\hline$i^{*}$ & 1.922912 & 0.037497 \\
\hline F814W & 1.803909 & 0.035176 \\
\hline 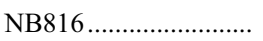 & 1.744951 & 0.034027 \\
\hline$z$ & 1.467711 & 0.028620 \\
\hline 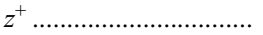 & 1.435914 & 0.028000 \\
\hline 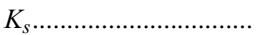 & 0.340677 & 0.006643 \\
\hline
\end{tabular}

TABLE 12

Photometric Offsets Measured from Other Surveys

\begin{tabular}{|c|c|c|}
\hline Filter & Offset to CFHT-LS & Offset to SDSS \\
\hline$u^{*}$ & 0.035 & $\ldots^{\mathrm{a}}$ \\
\hline$B_{\mathrm{J}} \ldots \ldots \ldots \ldots \ldots \ldots$ & 0.125 & 0.11 \\
\hline$g^{+} \ldots \ldots \ldots \ldots \ldots \ldots \ldots \ldots \ldots \ldots$ & -0.096 & -0.12 \\
\hline$V_{\mathrm{J}} \ldots \ldots \ldots \ldots \ldots \ldots \ldots \ldots \ldots$ & 0.040 & 0.03 \\
\hline$r^{+}$ & -0.080 & -0.07 \\
\hline 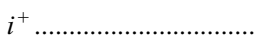 & -0.093 & -0.10 \\
\hline$z^{+}$ & -0.032 & -0.03 \\
\hline
\end{tabular}

${ }^{\text {a }}$ There is no linear relationship between $\operatorname{SDSS} u$ and CFHT $u^{*}$.

filter-dependent factor. These factors are given in Table 11 for filters used on the COSMOS field. These bandpass-dependent factors are calculated by integrating the filter response function against the Galactic extinction curve provided by Bolzonella et al. (2000) originally taken from Allen (1976).

\subsection{Absolute Photometric Zero-Point Corrections}

With typical overheads of 15 minutes per standard, it is extremely difficult to obtain a sufficient number of standard stars on Suprime-Cam. With three to five standards per band (Taniguchi et al. 2007), our standard-star calibrations are accurate to $\pm 0.05 \mathrm{mag}$ (see H. Aussel et al. 2007, in preparation). These offsets are larger than desired for accurate photometric redshifts.

Comparisons with the CFHT-LS and SDSS yield the zeropoint offsets given in Table 12. These were estimated by comparing total magnitudes for point sources between 21 and 24 mag for CFHT-LS, and 18 and 21 mag for SDSS converted to the COSMOS-AB photometric system. The color conversions between the surveys are given in Tables 8 and 9 and discussed further in H. Aussel et al. (2007, in preparation).

The zero-point corrections from the two surveys are consistent with one another and have an rms amplitude of $\pm 0.06 \mathrm{mag}$, which is slightly larger than the expected error. However, the zero points calculated in this way disagree with the ACS F $814 \mathrm{~W}$ photometry by $-0.118 \mathrm{mag}$ and fail to produce photometric redshifts free from systematic errors. Furthermore, Ilbert et al. (2006) find that the CFHT-LS zero points are inaccurate at the $0.05 \mathrm{mag}$ level.

Considering the number of present and ongoing observations of COSMOS, obtaining spectrophotometric standards in the field

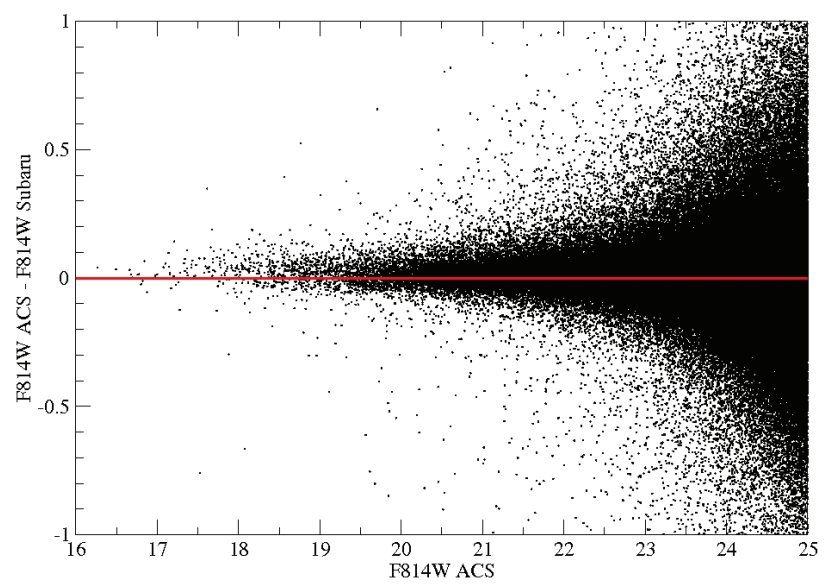

FIG. 10.-Offset between the ACS F814W-band photometry and the Subaru photometry as a function of magnitude after applying the offsets in Table 13. The Subaru $i^{+}$and $z^{+}$bands were used to estimate the F814W photometry. The scatter and zero-point error are within the expected error of the color conversion. 
TABLE 13

Photometric Offsets Calculated with Spectroscopic Redshifts

\begin{tabular}{|c|c|}
\hline Filter & Offset \\
\hline и & 0.0 \\
\hline$u^{*}$ & -0.084 \\
\hline$B_{\mathrm{J}}$ & 0.189 \\
\hline$g$ & 0.01 \\
\hline$g^{+}$ & -0.090 \\
\hline$V_{\mathrm{J}}$ & 0.04 \\
\hline$r$ & -0.033 \\
\hline$r^{+}$ & -0.040 \\
\hline$i$ & -0.037 \\
\hline$i^{+}$ & -0.020 \\
\hline$i^{*}$ & -0.005 \\
\hline NB816 ................................. & -0.072 \\
\hline F814W & 0.000 \\
\hline$z$ & -0.037 \\
\hline 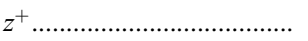 & 0.054 \\
\hline$K_{s} \ldots \ldots \ldots \ldots \ldots \ldots \ldots \ldots \ldots \ldots \ldots \ldots \ldots \ldots \ldots$ & $-0.097^{\mathrm{a}}$ \\
\hline
\end{tabular}

\footnotetext{
${ }^{\text {a }}$ Measured from 2MASS, not from
} spectroscopic redshifts. is a reasonable long-term solution to obtaining better quality photometry (Wolf et al. 2001). In the interim we are forced to rely on the existing calibrations and spectra of galaxies to recalibrate the photometric zero points for photometric redshifts.

These zero-point offsets are calculated by fitting the measured photometry, corrected for Galactic extinction, to galaxy templates at the known redshift and calculating the offset between the measured and expected photometry. To avoid systematic variations due to calibration errors in the galaxy templates, offsets between bands are calculated in rest-frame wavelength bins of $100 \AA$ separately for each template, then combined in a weighted average. This is effective because calibration errors in the template will create offsets which vary as a function of rest wavelength in the same way for all bands, while zero-point offsets between bands will be constant as a function of wavelength. Since these offsets are relative, the F814W is used as the reference for the absolute zero point (see Fig. 10). The offsets calculated using this method are given in Table 13.

This method is effective with a large sample of spectroscopic redshifts covering a large range in redshift along with photometry taken in many adjacent bands. For COSMOS there are insufficient data to correct the $K_{s}$ band due to the lack of photometric data between 0.9 and $2.2 \mu \mathrm{m}$. As a result, we rely on the $2 \mathrm{MASS}$ zero point for those data. Although the individual exposures were tied
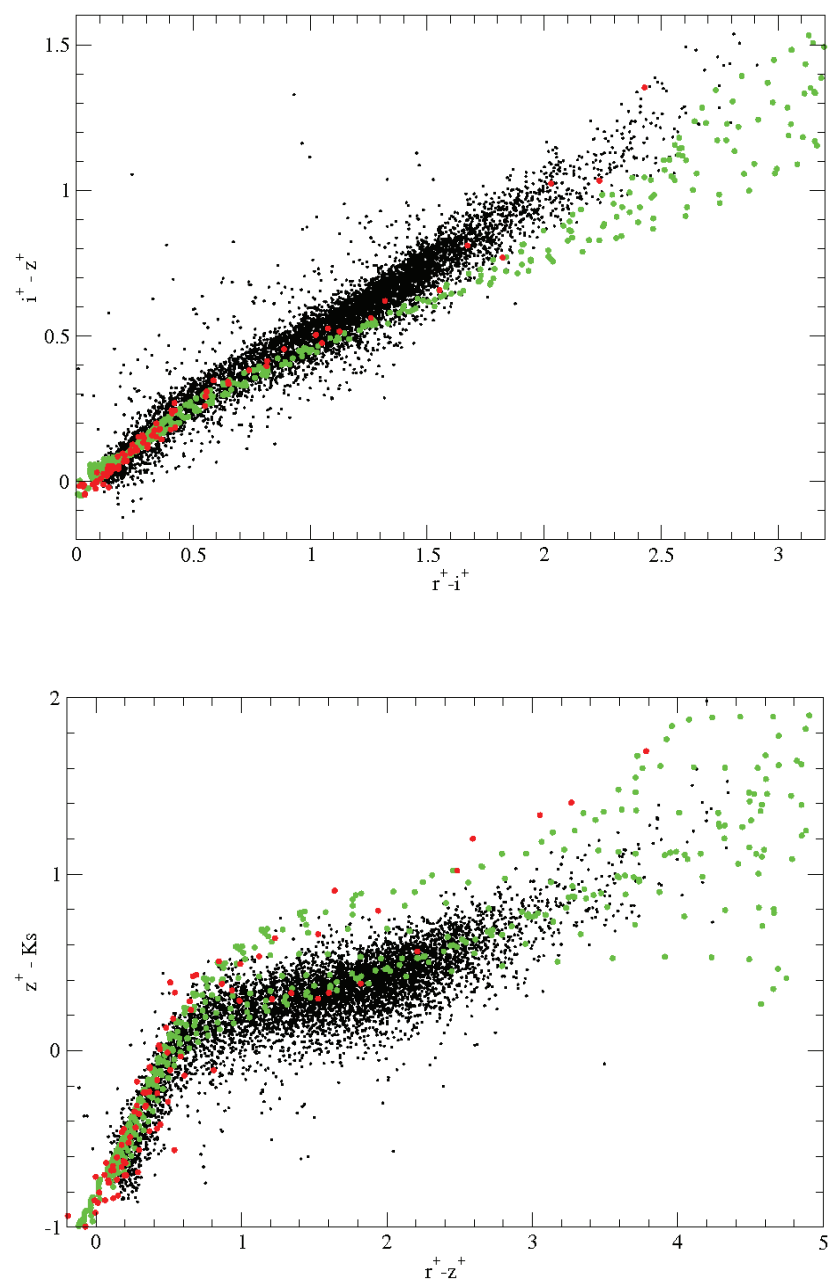

FIG. 11.-Four color-color plots for point sources in the COSMOS field (black), along with the expected colors from the BaSel, ver. 3.1 (Westera et al. 2002 ), and NextGen (Hauschildt \& Baron 2005; Brott \& Hauschildt 2005; Hauschildt et al. 2002) stellar libraries at the expected median metallicity of [Fe/H] $=-0.4$ (see Robin et al. 2007; green), and the Pickles (1998) spectral library $([\mathrm{Fe} / \mathrm{H}] \simeq 0$; red $)$. Notice that the Pickles library predicts systematically redder colors than the observations in the UV due to the high metallicity of these stars. 


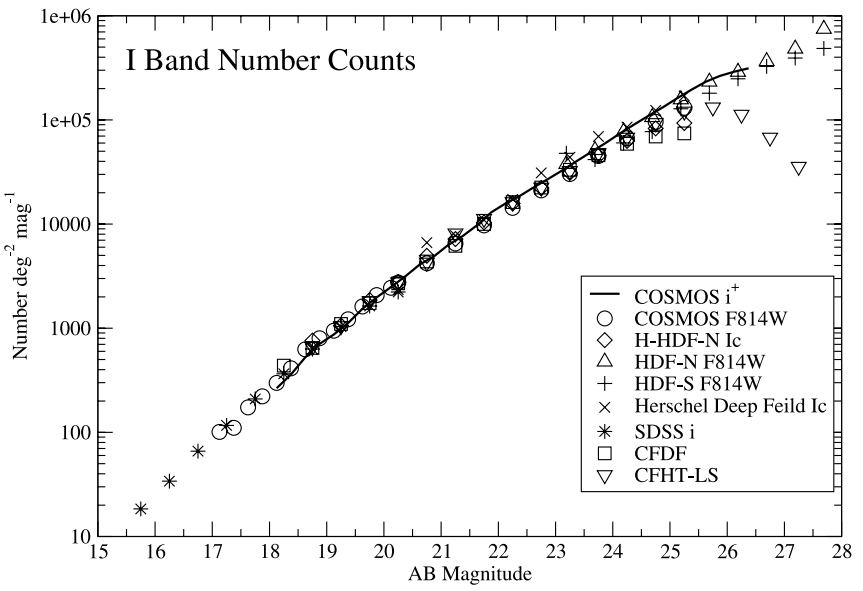

FIG. 12. - I-band number counts for the COSMOS ground-based catalog, the COSMOS F814W weak lensing catalog (Leauthaud et al. 2007), the HawaiiHubble Deep Field (H-HDF-N; Capak et al. 2004), the Hubble Deep Field-North (HDF-N; Williams et al. 1996; Metcalfe et al. 2001), the Hubble Deep FieldSouth (HDF-S; Metcalfe et al. 2001), the Herschel Deep Field (Metcalfe et al 2001), the SDSS (Yasuda et al. 2001), the Canada France Deep Fields (CFDFs; McCracken et al. 2003), and the Canada-France-Hawaii Telescope Legacy Survey (CFHT-LS; McCracken et al. 2007). Our counts are in good agreement with other surveys up to our $80 \%$ completeness limit at $i^{+}=26.5$.

to 2MASS, an offset of -0.097 mag is measured between the catalog photometry and the 2MASS catalog. This offset is likely an aperture correction between the photometry measured on the individual CTIO and KPNO exposures and the 2MASS catalog.

With the exception of the Suprime-Cam $B_{\mathrm{J}}$ band, which has known calibration problems (see H. Aussel et al. 2007, in preparation), the offsets are within the expected error of \pm 0.05 mag. After applying these offsets no systematic trend is measurable between photometric and spectroscopic redshifts for 842 objects with $0<z<1.2$. In addition, the $i^{+}$and $z^{+}$band agree with the F814W photometry to $0.007 \mathrm{mag}$, and the offsets between the COSMOS and CFHT-LS photometry agree with those in Ilbert et al. (2006) after applying color corrections. Furthermore, after applying the offsets the colors of stars agree with the predicted colors extremely well (see $\S 5.4$ ). These tests indicate that the zero points are within $0.01 \mathrm{mag}$ of the true $\mathrm{AB}$ zero points after applying these offsets.

These corrections were not applied to the released catalog, since they cannot be verified with external calibration sources at this time. However, we recommend applying the photometric redshift offsets for the best possible photometry and colors.

\subsection{Star Colors}

Stars are a good diagnostic of color accuracy because they form a tight sequence in most optical and near-IR color-color plots. Offsets as small as a few hundredths of a magnitude are visible when comparing expected and measured star colors. Furthermore, star colors are sensitive to the filter transmission profiles, providing a valuable check of the instrumental performance $(\mathrm{H}$. Aussel et al. 2007, in preparation).

Even at the resolution of $H S T$, compact galaxies and quasars contaminate a star selection based on morphology. These objects create scatter in color-color plots, obfuscating the stellar locus. The $B z K$ color-color diagram provides a much cleaner star selection (Daddi et al. 2004), and was used to select the objects plotted in Figure 11. The $B z K$ method is biased against faint blue stars due to the shallow $K_{s}$-band data; however, the effect is minimal since only objects with greater than $10 \sigma$ detections are plotted.
TABLE 14

$i^{+}$-Band Number Counts

\begin{tabular}{|c|c|c|}
\hline$i^{+} \mathrm{AB}$ & $\begin{array}{c}N \\
\left(\mathrm{deg}^{-2} \mathrm{mag}^{-1}\right)\end{array}$ & Poisson Error \\
\hline $15.625 \ldots$. & 6.534683 & 3.772801 \\
\hline 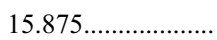 & 4.350995 & 3.076618 \\
\hline $16.125 \ldots \ldots \ldots \ldots \ldots \ldots$ & 4.345201 & 3.072521 \\
\hline $16.375 \ldots \ldots \ldots \ldots \ldots \ldots$ & 4.339069 & 3.068185 \\
\hline 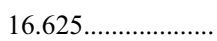 & 12.997791 & 5.306326 \\
\hline 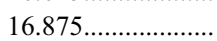 & 38.932047 & 9.176372 \\
\hline 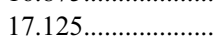 & 49.664184 & 10.355698 \\
\hline $17.375 \ldots \ldots \ldots$ & 75.444603 & 12.752466 \\
\hline 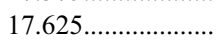 & 114.0366 & 15.664132 \\
\hline $17.875 \ldots \ldots \ldots \ldots \ldots \ldots \ldots$ & 154.62239 & 18.222423 \\
\hline 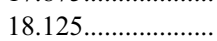 & 265.76389 & 23.866302 \\
\hline 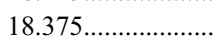 & 359.31933 & 27.722088 \\
\hline 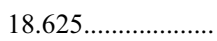 & 525.00773 & 33.473271 \\
\hline $18.875 \ldots \ldots \ldots \ldots \ldots \ldots \ldots$ & 706.95209 & 38.799037 \\
\hline $19.125 \ldots \ldots \ldots \ldots$ & 871.08483 & 43.019813 \\
\hline $19.375 \ldots \ldots \ldots \ldots \ldots$ & 1121.62 & 48.812246 \\
\hline $19.625 \ldots \ldots \ldots \ldots \ldots \ldots$ & 1538.1477 & 57.204325 \\
\hline 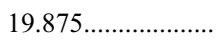 & 1978.0351 & 64.862326 \\
\hline 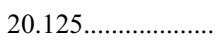 & 2487.0499 & 72.771793 \\
\hline $20.375 \ldots \ldots \ldots \ldots \ldots \ldots$ & 3150.2535 & 81.970061 \\
\hline $20.625 \ldots \ldots \ldots \ldots \ldots \ldots$ & 4084.8722 & 93.296944 \\
\hline $20.875 \ldots \ldots \ldots \ldots \ldots$ & 4900.1737 & 102.1979 \\
\hline $21.125 \ldots \ldots \ldots \ldots \ldots$ & 6309.9719 & 116.11697 \\
\hline 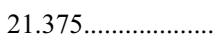 & 7656.9391 & 127.9895 \\
\hline $21.625 \ldots \ldots \ldots \ldots \ldots$ & 9710.6197 & 144.03897 \\
\hline $21.875 \ldots \ldots \ldots \ldots \ldots \ldots \ldots$ & 12951.105 & 166.68489 \\
\hline $22.125 \ldots \ldots \ldots \ldots \ldots \ldots$ & 15769.274 & 184.26274 \\
\hline $22.375 \ldots \ldots \ldots \ldots \ldots \ldots \ldots$ & 18613.365 & 199.97038 \\
\hline $22.625 \ldots \ldots \ldots \ldots \ldots$ & 22660.648 & 220.97698 \\
\hline $22.875 \ldots \ldots \ldots \ldots \ldots \ldots$ & 27245.578 & 242.54972 \\
\hline 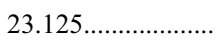 & 32943.681 & 267.97654 \\
\hline $23.375 \ldots \ldots \ldots \ldots \ldots \ldots$ & 40428.641 & 297.86588 \\
\hline $23.625 \ldots \ldots \ldots \ldots \ldots \ldots$ & 49326.475 & 328.82855 \\
\hline 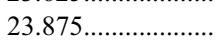 & 60011.205 & 363.35744 \\
\hline $24.125 \ldots \ldots \ldots \ldots \ldots$ & 74117.459 & 405.50413 \\
\hline 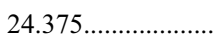 & 90498.908 & 450.39941 \\
\hline $24.625 \ldots \ldots \ldots \ldots \ldots$ & 109643.14 & 498.12066 \\
\hline $24.875 \ldots \ldots \ldots \ldots \ldots \ldots$ & 132088.39 & 548.23564 \\
\hline $25.125 \ldots \ldots \ldots \ldots \ldots \ldots$ & 161449.58 & 611.05628 \\
\hline $25.375 \ldots \ldots \ldots \ldots \ldots$ & 194924.55 & 678.02406 \\
\hline $25.625 \ldots \ldots \ldots \ldots \ldots \ldots$ & 232494.58 & 750.00984 \\
\hline $25.875 \ldots \ldots \ldots \ldots \ldots \ldots$ & 264905.22 & 808.54775 \\
\hline $26.125 \ldots \ldots \ldots \ldots \ldots \ldots$ & 290603.29 & 855.89733 \\
\hline $26.375 \ldots \ldots \ldots \ldots \ldots$ & 313097.56 & 910.75629 \\
\hline
\end{tabular}

Stellar libraries (e.g., Pickles 1998) typically contain solarmetallicity stars $([\mathrm{Fe} / \mathrm{H}] \simeq 0)$, which differ in color from the dominant subsolar-metallicity $([\mathrm{Fe} / \mathrm{H}] \simeq-0.4)$, thick-disk population in the COSMOS field (see Robin et al. 2007). This results in small offsets, especially in the ultraviolet where metal-line absorption will cause higher metallicity stars to appear redder.

Figure 11 shows four different color-color plots for stars along with colors for the Pickles (1998) library and a combination of the BaSel, version 3.1 (Westera et al. 2002), and NextGen theoretical libraries (Hauschildt \& Baron 2005; Brott \& Hauschildt 2005; Hauschildt et al. 2002) at $[\mathrm{Fe} / \mathrm{H}]=-0.4$. The star colors agree extremely well in all color-color plots, with an offset between the median expected and actual colors of less than $0.02 \mathrm{mag}$ in most bands. A correction of -0.05 mag is indicated by the stellar track for $u^{*}$, and systematic differences between the predicted and actual colors for $B_{\mathrm{J}}$ and $z^{+}$are observed. These are likely due to 


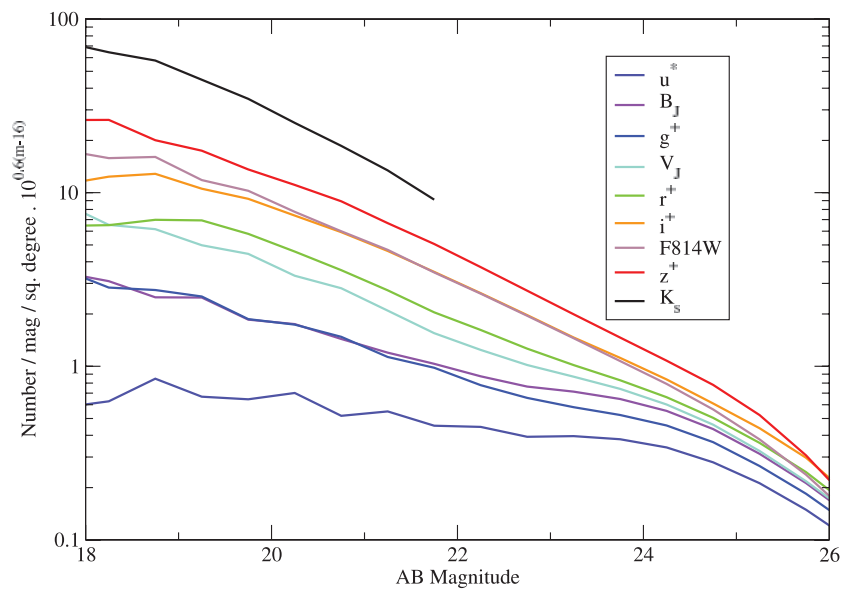

FIG. 13.-Number counts from the $i^{+}$-band-detected catalog for the $u^{*}, B_{\mathrm{J}}, g^{+}$, $V_{\mathrm{J}}, r^{+}, i^{+}, \mathrm{F} 814 \mathrm{~W}, z^{+}$, and $K_{s}$ bands divided by a Euclidian slope. The $u^{*}$ counts are nearly flat, while the $K_{s}$-band counts are very steep. A combination of a $\Omega_{m}=0.3, \Omega_{v}=0.7$ geometry, galaxy evolution, and redshifting is responsible for the band-dependent behavior.

our limited measurements of the filter throughput (H. Aussel et al. 2007, in preparation).

\subsection{Number Counts}

Differential number counts of galaxies are a simple but powerful measurement of the geometry of space, the evolution of the galaxy population, and the evolution of structure in the universe. They also provide a valuable check on data quality because they are sensitive to photometric calibration errors, detection completeness, and spurious detections. Figure 12 shows the $I$-band number counts from the COSMOS catalog compared to other surveys. The numbers are in good agreement; however, the COSMOS $i^{+}$-band counts are higher than the F814W ACS counts at magnitudes fainter than 24 . This is likely due to the lack of sensitivity at low surface brightness in ACS compared to Subaru and some contamination from spurious sources in the ground-based cata$\log$. The $i^{+}$-band galaxy number counts are given in Table 14; stars have been removed using a cut in ground-based FWHM since ACS does not cover the entire $2 \mathrm{deg}$.

Figure 13 shows the number counts for the COSMOS bands normalized to a slope of 0.6 , which is expected in a static Euclidian universe. In a universe with no galaxy evolution and a cosmology of $\Omega_{m}=0.3$ and $\Omega_{v}=0.7$, the number count slope should be below 0.6 in all bands. Furthermore, galaxies have a flat-to-red spectrum in units of $F_{\nu}$, so the $k$-correction is positive in $\mathrm{AB}$ magnitudes. This results in galaxies fading faster with redshift than they would from simple luminosity distance, further flattening the number count slope.

The slope of the normalized number counts in Figure 13 steepens with increasing wavelength, indicating that both the universe and the galaxy population are evolving. The UV luminosity of a typical galaxy increases with redshift due to increased star formation activity (Cowie et al. 1999; Lilly et al. 1996; Dahlen et al. 2005). This galaxy evolution counters the effects of an evolving universe, resulting in a $u^{*}$-band slope close to 0.6 . In the near-IR, there is little galaxy evolution (Dahlen et al. 2005), so the counts in the $K_{s}$ band are far below a slope of 0.6 .

\section{CONCLUSIONS}

Deep ground-based data were presented for the COSMOS field in 15 bands between 0.3 and $2.2 \mu \mathrm{m}$ along with an $i$-band- selected catalog of objects. We show that these data have the extremely high level of photometric consistency necessary for scientific pursuits such as large-scale structure studies. The expected zero-point variations are $<1 \%$ across the field; this level of photometry was achieved by constructing flat fields directly from object fluxes rather than using sky or dome flats.

Variations in the point-spread function (PSF) are a significant source of uncertainty in ground-based photometry. This is due to the fact that the non-Gaussian portion of the PSF is difficult to match across multiple bands, resulting in 2\%-5\% errors in color measurement if uncorrected. The variation was minimized by adjusting the Gaussian filter used for PSF matching to provide the same fraction of light to fall in a $3^{\prime \prime}$ aperture in all bands. However, this means that only the $3^{\prime \prime}$ aperture photometry is reliable for colors. Photometry measured in other aperture sizes should be corrected for variations in the PSF.

An $i^{+}$-band selection was used for the multiband catalog after analyzing the tradeoffs between a $\chi^{2}$ and $i$-band-detected cata$\log$. The decrease in resolution and increased problems with a $\chi^{2}$ catalog outweigh the benefits of increased sensitivity and panchromatic completeness. The resulting catalog is $91 \%$ complete at $i^{+}=25.0,87 \%$ complete at $i^{+}=26.0$, and $50 \%$ complete at $i^{+}=27.4$.

Our photometric zero points measured from standard stars (H. Aussel et al. 2007, in preparation) are accurate to 5\%. These zero-point offsets can be significantly improved by using galaxies with known redshifts to adjust the zero-point calibration. The corrected zero points appear accurate to better than $2 \%$ based on star colors; this will be verified with future internal spectrophotometric standards.

Number counts measured from the $i^{+}$-band-selected catalog agree well with previous studies. The effects of galaxy evolution and an expanding universe are clearly visible in the panchromatic counts, demonstrating the importance of multicolor surveys.

We would like to thank the COSMOS team (http://cosmos .astro.caltech.edu), the staff at Caltech, CFHT, CTIO, KPNO, NAOJ, STSCI, TERAPIX, and the University of Hawaii for supporting this work and making it possible. Support for this work was provided by NASA grant HST-GO-09822 and NSF grant OISE-0456439. FLAMINGOS was designed and constructed by the IR instrumentation group (PI: R. Elston) at the University of Florida, Department of Astronomy, with support from NSF grant AST 97-31180 and Kitt Peak National Observatory. Funding for the Sloan Digital Sky Survey (SDSS) has been provided by the Alfred P. Sloan Foundation, the Participating Institutions, the National Aeronautics and Space Administration, the National Science Foundation, the US Department of Energy, the Japanese Monbukagakusho, and the Max Planck Society. The SDSS Web site is at http://www.sdss.org. The SDSS is managed by the Astrophysical Research Consortium for the Participating Institutions. The Participating Institutions are the University of Chicago, Fermilab, the Institute for Advanced Study, the Japan Participation Group, the Johns Hopkins University, Los Alamos National Laboratory, the Max Planck Institute for Astronomy, the Max Planck Institute for Astrophysics, New Mexico State University, the University of Pittsburgh, Princeton University, the United States Naval Observatory, and the University of Washington. D. T. acknowledges support from NASA through a Long Term Space Astrophysics grant (NAG5-10955, NRA-00-01-LTSA-064). This work is based (in part) on data products produced at the TERAPIX data center located at the Institut d'Astrophysique de Paris. 
Abazajian, K., et al. 2004, AJ, 128, 502

Adelberger, K. L., Steidel, C. C., Shapley, A. E., Hunt, M. P., Erb, D. K., Reddy, N. A., \& Pettini, M. 2004, ApJ, 607, 226

Aguirre, J. E., et al. 2007, ApJS, in press

Allen, C. W. 1976, Astrophysical Quantities (3rd ed.; London: Athlone Press)

Aune, S., et al. 2003, Proc. SPIE, 4841, 513

Bertin, E., \& Arnouts, S. 1996, A\&AS, 117, 393

Bertoldi, F., et al. 2007, ApJS, in press

Bolzonella, M., Miralles, J.-M., \& Pelló, R. 2000, A\&A, 363, 476

Boulade, O., et al. 2003, Proc. SPIE, 4841, 72

Brott, I., \& Hauschildt, P. H. 2005, in Proc. Gaia Symp., The Three-Dimensional Universe with Gaia, ed. C. Turon, K. S. O'Flaherty, \& M. A. C. Perryman (ESA SP-576; Noordwijk: ESA), 565

Capak, P., et al. 2004, AJ, 127, 180

Casertano, S., et al. 2000, AJ, 120, 2747

Cowie, L. L., Songaila, A., \& Barger, A. J. 1999, AJ, 118, 603

Daddi, E., Cimatti, A., Renzini, A., Fontana, A., Mignoli, M., Pozzetti, L., Tozzi, P., \& Zamorani, G. 2004, ApJ, 617, 746

Dahlen, T., Mobasher, B., Somerville, R. S., Moustakas, L. A., Dickinson, M. Ferguson, H. C., \& Giavalisco, M. 2005, ApJ, 631, 126

Elston, R. 1998, Proc. SPIE, 3354, 404

Franx, M., et al. 2003, ApJ, 587, L79

Giavalisco, M., et al. 2004, ApJ, 600, L93

Hasinger, G., et al. 2007, ApJS, 172, 29

Hauschildt, P. H., Allard, F., Schweitzer, A., \& Baron, E. 2002, in ASP Conf. Ser. 274, Observed HR Diagrams and Stellar Evolution, ed. T. Lejeune \& J. Fernandes (San Francisco: ASP), 95

Hauschildt, P., \& Baron, E. 2005, Mem. Soc. Astron. Italiana Suppl., 7, 140

Ilbert, O., et al. 2007, A\&A, 457, 841

Koekemoer, A., et al. 2007, ApJS, 172, 196

Komiyama, Y., et al. 2003, Proc. SPIE, 4841, 152

Landolt, A. U. 1992, AJ, 104, 340

Leauthaud, A., Massey, R., Rhodes, J., \& Capak, P. L. 2007, ApJS, 172, 219

Lilly, S. J., Le Fevre, O., Crampton, D., Hammer, F., \& Tresse, L. 1995, ApJ, 455,50
REFERENCES

Lilly, S. J., Le Fevre, O., Hammer, F., \& Crampton, D. 1996, ApJ, 460, L1 Magnier, E. A., \& Cuillandre, J.-C. 2004, PASP, 116, 449

McCracken, H. J., Aussel, H., Capak, P., El-Zant, A., Guzzo, L., \& Scoville, N. Z. 2007, ApJS, 172, 314

McCracken, H. J., et al. 2003, A\&A, 410, 17

Metcalfe, N., Shanks, T., Campos, A., McCracken, H. J., \& Fong, R. 2001, MNRAS, 323, 795

Monet, D. G., et al. 2003, AJ, 125, 984

Pickles, A. J. 1998, PASP, 110, 863

Probst, R. G., et al. 2003, Proc. SPIE, 4841, 411

Rix, H.-W., et al. 2004, ApJS, 152, 163

Robin, A., et al. 2007, ApJS, 172, 545

Sanders, D. B., et al. 2007, ApJS, 172, 86

Schinnerer, E., et al. 2004, AJ, 128, 1974 2007, ApJS, 172, 46

Schlegel, D. J., Finkbeiner, D. P., \& Davis, M. 1998, ApJ, 500, 525

Scoville, N. Z., et al. 2007a, ApJS, 172, 38 2007b, ApJS, 172, 1

Skrutskie, M. F., et al. 2006, AJ, 131, 1163

Steidel, C. C., Adelberger, K. L., Giavalisco, M., Dickinson, M., \& Pettini, M. 1999, ApJ, 519, 1

Steidel, C. C., Adelberger, K. L., Shapley, A. E., Pettini, M., Dickinson, M., \& Giavalisco, M. 2003, ApJ, 592, 728

Steidel, C. C., Giavalisco, M., Dickinson, M., \& Adelberger, K. L. 1996, AJ, 112,352

Taniguchi, Y., Scoville, N. Z., Ajiki, M., Sanders, D. B., Mobasher, B., Aussel, H., \& Capak, P. L. 2007, ApJS, 172, 9

Westera, P., Lejeune, T., Buser, R., Cuisinier, F., \& Bruzual, G. 2002, A\&A, 381,524

Williams, R. E., et al. 1996, AJ, 112, 1335

Wolf, C., et al. 2001, A\&A, 365, 681

Yasuda, N., et al. 2001, AJ, 122, 1104

Zamojski, M. A., et al. 2007, ApJS, 172, 468 